\title{
In vivo antileishmanial activity and histopathological evaluation in Leishmania infantum infected hamsters after treatment with a furoxan derivative
}

\author{
Letícia de Almeida ${ }^{a}$, Thaís Gaban Passalacqua ${ }^{a}$, Luiz Antonio Dutra ${ }^{a}$, \\ Jéssica N. Varonez da Fonseca ${ }^{a}$, Rhayanne F. Queiroz Nascimento ${ }^{a}$, Kely Braga Imamura ${ }^{a}$, \\ Cleverton Roberto de Andrade ${ }^{\mathrm{b}}$, Jean Leandro dos Santos ${ }^{\mathrm{a}}$, Márcia A.S. Graminha ${ }^{\mathrm{a} \text {,* }}$ \\ a Universidade Estadual Paulista (UNESP), Faculdade de Ciências Farmacêticas, Câmpus Araraquara, Brazil \\ b Universidade Estadual Paulista (UNESP), Faculdade de Odontologia, Câmpus Araraquara, Brazil
}

\section{A R T I C L E I N F O}

\section{Keywords:}

Visceral leishmaniasis

In vivo antileishmanial activity

L. infantum

Semi-quantitative histopathological analysis

NO-donor

Furoxan derivatives

\begin{abstract}
A B S T R A C T
$\mathrm{N}$-oxide derivatives compounds such as furoxan and benzofuroxan are promising scaffolds for designing of new antileishmanial drugs. A series of furoxan (1,2,5-oxadiazole 2- $N$-oxide) (compounds 4a-b, and 14a-f) and benzofuroxan (benzo[c][1,2,5] oxadiazole1- $N$-oxide) (compounds 8a-c) derivatives were evaluated against in vitro cultured $L$. infantum promastigotes and amastigotes. The compounds exhibited activity against promastigote and intracellular amastigote forms with $\mathrm{EC}_{50}$ values ranging from 2.9 to $71.2 \mu \mathrm{M}$ and 2.1 to $18.2 \mu \mathrm{M}$, respectively. The most promising compound, $14 \mathrm{e}$, showed good antileishmanial activity $\left(\mathrm{EC}_{50}=3.1 \mu \mathrm{M}\right)$ against intracellular amastigote forms of $L$. infantum with a selectivity index, based on murine macrophages $(S I=66.4)$, almost 3-times superior to that presented by the standard drug amphotericin B (AmpB). The efficacy of 14e to eliminate the parasites in vivo was also demonstrated. Treatment of L. infantum-infected hamsters with compound $14 \mathrm{e}$ at $3.0 \mathrm{mg} / \mathrm{Kg}$ /day led to a meaningful reduction of parasite load in spleen (49.9\%) and liver (54.2\%), respectively; these data were corroborated by histopathological analysis, which also revealed reduction in the number of inflammatory cells in the liver of the treated animals. Moreover, histological analysis of the spleen and kidney of treated animals did not reveal alterations suggestive of toxic effects. The parasite load reduction might be related to NO production, since this molecule is a NO-donor. We observed neither side effects nor elevation of hepatic/renal biomarker levels in the plasma. The data herein presented suggest that the compound should be considered in the development of new drugs for treatment of visceral leishmaniasis.
\end{abstract}

\section{Introduction}

Leishmaniasis is a neglected tropical disease caused by parasites of the genus Leishmania. This disease is endemic in 98 countries spread throughout Asia, Africa, South and Central America and Southern Europe $[1,2]$. The annual incidence of leishmaniasis is 600,000 human infections, and the prevalence is 12 million cases [3,4]. Leishmaniasis is often considered a complex of diseases that present two major manifestations, the visceral (VL) and the cutaneous forms (CL). Moreover, they present a number of other manifestations including the mucocutaneous (MCL), the diffuse (DCL) and the post-kalazar (PKDL) leishmaniasis. Importantly, the clinical presentation of leishmaniasis is dependent upon both the parasite species and the host's immune system [5]. For CL, MCL or DCL, the symptoms remain localized to the skin or mucous membranes. In contrast, VL disseminates to internal organs such as liver, spleen and bone marrow, which is fatal if left untreated. The proliferation of parasites within macrophages present in those organs causes progressive hepatosplenomegaly as well as bone marrow suppression. If not treated, patients might develop pancytopenia and immunosuppression which render them susceptible to other infections causing patient death [2].

Antileishmanial drugs remain the most important tool for the treatment and control of both VL and CL [4]. The first-choice treatment is based on the use of the old-fashioned pentavalent antimonials, sodium stibogluconate and meglumine antimoniate [6]. These drugs are highly toxic and their administration is painful and requires long-term treatment [7] which often lead to patients abandoning the treatment with consequent increase in the number of circulating drug-resistant

\footnotetext{
* Corresponding author.

E-mail address: graminha@fcfar.unesp.br (M.A.S. Graminha).
} 
strains [8]. Amphotericin B (AmpB) (and its liposomal formulations), pentamidine, paromomycin and miltefosine are currently being used in leishmaniasis treatment as second line therapies, however they also show side effects [9].

In the past 10 years, progress has been made to the discovery of new alternative treatments [10-12]. Among the potential new chemical entities, furoxan (1,2,5-oxadiazole 2 - $N$-oxide) and benzofuroxan (benzo [c][1,2,5] oxadiazole 1 - $N$-oxide) derivatives have been explored as pharmacophores to design antileishmanial candidates [13,14]. Boiani and collaborators characterized furoxans that showed anti-Kinetoplastea properties in in vitro assays [15]. Recently, Hernandez and colleagues described the anti-Leishmania and anti-T. cruzi activity of furoxanyl $\mathrm{N}$-acylhydrazones derivatives presenting high selectivity [16]. We have previously reported the synthesis of furoxan and benzofuroxan derivatives containing the $\mathrm{N}$-acylhydrazone subunit and their nitric oxide (NO) donor ability as well as their anti-L. amazonensis activity [17].

Herein we describe the in vitro anti-L. infantum activity of furoxan (compounds 4a-b and 14a-f) and benzofuroxan (compounds 8a-c) derivatives as well as the in vivo evaluation of the most promising furoxan derivative named 14e.

\section{Materials and methods}

\subsection{Animals}

Male Swiss albino mice six to eight weeks old were obtained from breeding stocks of the Universidade Estadual Paulista (UNESP, Araraquara, São Paulo, Brazil). Eight-week-old male Golden hamsters (Mesocricetus auratus) were acquired from Anilab (São Paulo, Brazil). All animals were maintained at the Universidade Estadual Paulista (UNESP, Araraquara, São Paulo, Brazil) in single-sex cages under a 12-h light/12-h dark cycle (lights on at 06:00 am) in a controlled-temperature room $\left(22 \pm 2{ }^{\circ} \mathrm{C}\right)$ and they were fed ad libitum. This study was approved by the Ethics Committee for Animal Experimentation of the Faculdade de Ciências Farmacêuticas, UNESP (CEUA/FCF/CAr n ${ }^{\circ}$ 09/ 2013 and CEUA/FCF/CAr $n^{\circ}$ 23/2014) in agreement with the guidelines of the Sociedade Brasileira de Ciência de Animais de Laboratório (SBCAL) and of the Conselho Nacional de Controle da Experimentação Animal (CONCEA).

\subsection{Parasites}

Promastigotes of $L$. infantum strain MHOM/BR/1972/LD, kindly provided by Dr. José A. Lindoso from the Instituto de Medicina Tropical (São Paulo, Brazil), were mantained in Schneider's medium, supplemented with $10 \%$ heat-inactivated fetal calf serum (hiFCS), 10\% male human urine, $1 \%$ penicilin/streptomycin (Pen/Strep).

\subsection{Furoxan and benzofuroxan derivatives}

Furoxan and benzofuroxan derivatives were synthesized as previously described [17]. The purified products were: 2-amino$N=-$ [(1E)-(2-oxido-4-phenyl-1,2,5-oxadiazol-3-yl) - [(1E)-

(2-oxido-4-phenyl-1,2,5-oxadiazol-3-yl) methylene]benzohydrazide (compound 4a); 4-amino- $N=-[(1 \mathrm{E})$-(2-oxido-4-phenyl-1,2,5-oxadiazol-3-yl) - [(1E)-(2-oxido-4-phenyl-1,2,5-oxadiazol-3-yl) methylene]benzohydrazide (compound $4 \mathrm{~b}$ ); 2 -amino- $\mathrm{N}=-[(1 \mathrm{E})$-(3-oxido2,1,3-benzoxadiazol-5-yl) - [(1E)-(3-oxido-2,1,3-benzoxadiazol-5-yl) methylene]benzohydrazide (compound 8a); 3-amino- $N=-[(1 \mathrm{E})-(3-$ oxido-2,1,3-benzoxadiazol-5-yl) - [(1E)-(3-oxido-2,1,3-benzoxadiazol5-yl) methylene]benzohydrazide (compound $\mathbf{8 b}$ ); 4-amino$N=-$ [(1E)-(3-oxido-2,1,3-benzoxadiazol-5-yl) - [(1E)-(3-oxido-2,1,3benzoxadiazol-5-yl) methylene]benzohydrazide (compound 8c); 2-hydroxy- $N=-$ [(1E)-(3-\{[5-oxido-4-(phenylsulfonyl)-1,2,5oxadiazol-3yl]oxy\}phenyl)methylene]benzohydrazide $-[(1 E)-(3-\{[5$-oxido-4- (phenylsulfonyl)-1,2,5oxadiazol-3-yl]oxy\}phenyl)methylene]benzohydrazide (compound 14a); 3-hydroxy- $N=-[$ (1E)-(3-\{[5-oxido-4-(phenylsulfonyl)-1,2,5oxadiazol-3-yl] oxy\}phenyl)methylene]benzohydrazide - [(1E)-(3-\{[5-oxido-4-(phenylsulfonyl)-1,2,5oxadiazol-3-yl] oxy\}phenyl)methylene]benzohydrazide (compound 14b); 4-hydroxy$N=-$ [(1E)-(3-\{[5-oxido-4-(phenylsulfonyl)-1,2,5oxadiazol-3-yl]oxy $\}$ phenyl)methylene]benzohydrazide - [(1E)-(3-\{[5-oxido-4-(phenylsulfonyl)-1,2,5oxadiazol-3-yl] oxy\}phenyl)methylene]benzohydrazide (compound 14c); 2-hydroxy- $N=-[(1 \mathrm{E})-(4-\{[5-o x i d o-4-(p h e-$ nylsulfonyl)-1,2,5oxadiazol-3-yl] oxy\}phenyl)methylene]benzohydrazide - [(1E)-(4-\{[5-oxido-4-(phenylsulfonyl)-1,2,5oxadiazol-3-yl] oxy\}phenyl)methylene]benzohydrazide (compound 14d); 3-hydroxy$N=-$ [(1E)-(4-\{[5-oxido-4-(phenylsulfonyl)-1,2,5oxadiazol-3-yl $]$ oxy $\}$ phenyl)methylene]benzohydrazide - [(1E)-(4-\{[5-oxido-4-(phenylsulfonyl)-1,2,5oxadiazol-3-yl] oxy\}phenyl)methylene]benzohydrazide (compound 14e); 4-hydroxy- $N=-[(1 \mathrm{E})$-(4-\{[5-oxido-4-(phenylsulfonyl)-1,2,5oxadiazol-3-yl] oxy\}phenyl)methylene]benzohydrazide - [(1E)-(4-\{[5-oxido-4-(phenylsulfonyl)-1,2,5oxadiazol-3-yl] oxy\}phenyl)methylene]benzohydrazide (compound 14f). Stock solutions were prepared in dimethylsulfoxide (DMSO) for use in the in vitro assays.

\subsection{In vitro efficacy of furoxan and benzofuroxan derivatives against $L$. infantum promastigotes}

Cultured promastigotes of $L$. infantum in the mid-log phase growth phase were seeded at $1 \times 10^{7}$ parasites/mL in 96-well flat-bottom plates (TPP; Sigma-Aldrich). The tested compounds dissolved in DMSO (the highest concentration was $3 \%$, which was not hazardous to the parasites, as determined prior to the assays) and AmpB in sterile water were added to the parasite suspensions to final concentrations between 0.5 and $100 \mu \mathrm{M}$, and incubated at $28^{\circ} \mathrm{C}$ for $72 \mathrm{~h}$. Leishmanicidal effects were assessed by the 3-[4,5-dimethylthiazol-2-yl]-2,5-diphenyl-2H-tetrazolium bromide (MTT) method [11]. Absorbances were read in a 96well plate reader (Robonik) at $490 \mathrm{~nm}$. The concentration that caused a $50 \%$ decrease in parasite viability compared to the control was determined by non-linear regression analysis and expressed as the effective concentration $\left(\mathrm{EC}_{50-\mathrm{PRO}}\right)$ in $\mu \mathrm{M}[11,17]$. The assays were carried out in biological and experimental triplicates. The data were expressed as average \pm SD from three independent experiments.

\subsection{Cytotoxicity assays}

Murine peritoneal macrophages were collected after thioglycolatestimulation. The cells were seeded in 96-well flat-bottom plates (TPP) at a density of $3 \times 10^{5}$ cells/well $(100 \mu \mathrm{L} /$ well) in RPMI-1640 medium supplemented with $10 \%$ hiFCS, $25 \mathrm{mM}$ HEPES, and $2 \mathrm{mM}$ L-glutamine and Pen/Strep $1 \%$, and incubated for $4 \mathrm{~h}$ at $37^{\circ} \mathrm{C}$ in a $5 \% \mathrm{CO}_{2}$-air mixture to adhere. The medium was removed, and then new medium was added to the cells, which were treated with different concentrations of compounds and AmpB ( 4 to $400 \mu \mathrm{M}$ ). Cells without drugs were used as negative control. After that, plates were incubated for $24 \mathrm{~h}$ at $37^{\circ} \mathrm{C}$ in a $5 \% \mathrm{CO}_{2}$-air mixture. Subsequently, the MTT colorimetric assay was carried out as described above. Absorbance was read in a 96-well plate reader (Robonik) at $595 \mathrm{~nm}$. The drug concentration corresponding to $50 \%$ of cell growth inhibition was expressed as the cytotoxic concentration $\left(\mathrm{CC}_{50}\right)$ in $\mu \mathrm{M}$ [17]. The cytotoxicity for macrophages and parasites were compared using the selectivity index (SI), which was determined as the ratio between $\mathrm{CC}_{50}$ for macrophages and $\mathrm{EC}_{50}$ for $L$. (L.) infantum.

2.6. Leishmanicidal efficacy of furoxan and benzofuroxan derivatives on $L$. infantum-infected macrophages

The activity of furoxan and benzofuroxan derivatives (4a, 8a-c, 14a-e) against intracellular amastigotes was evaluated in mouse 
peritoneal macrophages infected with $L$. infantum. Murine macrophages were collected from the peritoneal cavity of Swiss mice after thioglycolate-stimulation and plated at $3 \times 10^{5}$ cells/well on coverslips (13$\mathrm{mm}$ diameter), previously arranged in a 24-well plate containing RPMI1640 medium supplemented with $10 \%$ of hiFCS, $25 \mathrm{mM}$ HEPES, $2 \mathrm{mM}$ L-glutamine and 1\% Pen/Strep, and allowed to adhere for $4 \mathrm{~h}$ at $37{ }^{\circ} \mathrm{C}$ in $5 \% \mathrm{CO}_{2}$. Adherent macrophages were infected with promastigotes forms in the stationary growth phase using a ratio of 10:1 parasites per macrophage at $37{ }^{\circ} \mathrm{C}$ in $5 \% \mathrm{CO}_{2}$ for $20 \mathrm{~h}$ to allow parasite multiplication. The infected cells were then treated with different concentrations of each compounds and AmpB $(0.6$ to $20 \mu \mathrm{M})$ for $24 \mathrm{~h}$. After incubation, the cells were fixed with methanol, Giemsa stained and examined by light microscopy. The number of amastigotes/100 macrophage cells and the percentage of infected cells were determined. The concentration that caused $50 \%$ growth inhibition compared to the control was determined by non-linear regression analysis and expressed as the effective concentration ( $\mathrm{EC}_{50-\mathrm{AMA}}$ ) in $\mu \mathrm{M}$ [17]. The data were expressed as average \pm SD from three independent experiments.

\subsection{In vitro nitric oxide production}

Culture supernatants from murine peritoneal macrophages infected with $L$. infantum and treated with compounds 4a, 8a-c, 14a-e at $10 \mu \mathrm{g}$ / $\mathrm{mL}$ were analysed. Culture supernatant from non-infected macrophages was used as negative control, while macrophages infected with $L$. infantum and treated with $10 \mu \mathrm{g} / \mathrm{mL}$ of lipopolysaccharide (LPS) served as positive control. The supernatants were collected after $48 \mathrm{~h}$ treatment and the nitrite production was determined using Griess' reagent [18]. A mixture of equal parts of culture supernatants and Griess' reagent was pipetted into 96-well plates to a total volume of $200 \mu \mathrm{L}$, followed by incubation at room temperature for $10 \mathrm{~min}$. The absorbance was read in a 96-well plate reader (Robonik) at $540 \mathrm{~nm}$ [19]. The results were determined after extrapolation of values obtained from a standard curve constructed using sodium nitrite $\left(\mathrm{NaNO}_{2}\right)$ and expressed as micromolar concentrations. All assays were performed in duplicate and the statistical analyses were perfomed by Tukey's test $(P<0.05)$ [17].

\subsection{In vivo leishmanicidal activity}

In order to evaluate in vivo leishmanicidal activity of 14e, eightweek-old male Golden hamsters were randomly separated in seven groups containing five animals each. The animals were intraperitonealy infected with $1 \times 10^{8}$ of $L$. infantum promastigotes in the late exponential growth phase. Seventy-five days post-infection, i.e., when the animals presented clinical signs of the disease; such as increased abdominal size that indicates hepatosplenomegaly, hair loss accompanied by ulcers localized in the snout; the infected animals received daily intraperitoneal doses of $14 \mathrm{e}(3,1.5$ or $0.75 \mathrm{mg} / \mathrm{Kg} / \mathrm{day})$, the reference drug AmpB $(20 \mathrm{mg} / \mathrm{Kg} /$ day - diluted in sterile water according to the manufacturer's instructions) or the vehicle for 15 days; additionally, untreated infected animals and non-infected animals were also evaluated. At the end of the treatment, the animals were euthanized and the parasite burden in the spleen and the liver was determined by limiting dilution methodology $[20,21]$. The data are expressed as average \pm SD.*: Statistical significance of the difference relative to the untreated infected group $(\mathrm{P}<0.05)$ was determined by Tukey's test.

\subsection{Toxicity assays for golden hamsters}

Serum concentrations of alanine (ALT) and aspartate (AST) aminotransferases, alkaline phosphatase (ALP) and creatinine at the end of the treatments were determined in Golden hamsters by dry chemistry on a STAT Analyzer (Ortho Clinical Vitros 250 Chemistry System) and compared with reference values for hamsters. The data are expressed as average \pm SD. *: statistical significance of the difference relative to the non-infected animals (healthy animals) $(P<0.05)$; $\alpha$ : statistical
Table 1

Numerical criteria for the classification of inflammatory infiltrates according to the cellular types found.

\begin{tabular}{|c|c|c|c|c|c|}
\hline \multirow[t]{2}{*}{ Inflammatory parameters } & \multicolumn{4}{|c|}{ Intensity } & \multirow[t]{2}{*}{ Type } \\
\hline & $\begin{array}{l}\text { Absent } \\
(0 \%)\end{array}$ & $\begin{array}{l}\text { Mild } \\
(1-33 \%)\end{array}$ & $\begin{array}{l}\text { Moderate } \\
(34-66 \%)\end{array}$ & $\begin{array}{l}\text { Intense } \\
(>66 \%)\end{array}$ & \\
\hline Neutrophils & 0 & -1 & -2 & -3 & $\begin{array}{l}\text { Acute } \\
(-6 \text { to } \\
-3)\end{array}$ \\
\hline Interstitial edema & 0 & -1 & -2 & -3 & $\begin{array}{l}\text { Mixed } \\
\text { (-3.1 to } \\
3\end{array}$ \\
\hline Lymphomononuclear & 0 & 1 & 2 & 3 & Chronic \\
\hline Granulomes & 0 & 1 & 2 & 3 & $\begin{array}{l}\text { (3.1 to } \\
6)\end{array}$ \\
\hline
\end{tabular}

significance of the difference relative to the untreated infected animals $(P<0.05)$ were determined by Tukey's test.

\subsection{Histopathological analysis of golden hamsters}

All hamsters were euthanized at the end of the treatment and parts of the liver, spleen and kidney were fixed in formaline, paraffin sectioned and stained with Haematoxylin and Eosin (H\&E). Slidemounted sections were studied under a microscope using appropriate magnifications. The histopathological analyses for the experimental groups were blindly performed by a pathologist. Each glass slide was systematically analyzed.

Analysis of the liver involved hepatocytes morphology, necrosis, edema and the presence of inflammatory infiltrates in the periportal region, as well as the identification of amastigotes and the presence of Schaumann's bodies in the tissue. The inflammatory infiltrates near to periportal spaces were quantified and classified according to type (acute, chronic or mixed) and intensity (mild, moderate or intense) (Table 1). At the lobular region the inflammatory infiltrates were quantified and classified by type (acute, chronic and mixed) (Table 1) and size as small (up to 25 cells) or large (more than 25 cells). Quantitative analysis of inflammatory infiltrates in the liver was performed. The data are expressed as average \pm SD.*: Statistical significance of the difference relative to the untreated infected group $(\mathrm{P}<0.05)$ was determined by Tukey's test.

The spleen analyses involved the presence of capsule, white/red pulp, marginal zone and follicules in this organ. The presence of granulomes and amastigotes were also evaluated. For the kidney, the organ structure in the cortical and medular areas was analysed. In the cortical area, the analysis comprised the presence of Bowman's capsule, the Bowman's capsule space, and the proximal and distal ducts; in the medular regions, the ducts and the presence of inflammation were examined. The analyses of red/white pulp, marginal zone and Bowman's capsule and Bowman's capsule space, proximal/distal ducts were made through semi-quantitative analysis

\section{Results}

\subsection{Efficacy of furoxan and benzofuroxan derivatives against $L$. infantum} promastigotes

The antileishmanial activity of furoxan (compounds $\mathbf{4 a}$ and $-\mathbf{b}$, and 14a to $-\mathbf{f}$ ) and benzofuroxan (compounds 8a to $-\mathbf{c}$ ) derivatives was initially determined against the insect stage of $L$. infantum (Table 2). The furoxan derivatives (14b-14f) showed variable activities against the promastigotes ranging from $5.7 \mu \mathrm{M}$ to $9.8 \mu \mathrm{M}$, while the selectivity index showed that $14 \mathrm{e}\left(\mathrm{SI}_{1}=21.3\right)$ is the less cytotoxic molecule for mammalian cells among this group of derivatives. The remaining compound 14a has neither shown good anti-promastigote activity 
Table 2

Biological activity of furoxan/benzofuroxan derivatives and amphotericin B (AmpB) against promastigotes and amastigotes of $L$. infantum $\left(\mathrm{EC}_{50}\right)$ and cytotoxicity against murine macrophages $\left(\mathrm{CC}_{50}\right)$. The results are expressed in $\mu \mathrm{M}$.

\begin{tabular}{|c|c|c|c|c|c|}
\hline \multirow[t]{2}{*}{ Compounds } & \multirow{2}{*}{$\begin{array}{l}\text { Macrophages } \\
{ }^{\mathrm{a}} \mathrm{CC}_{50}\end{array}$} & \multicolumn{2}{|c|}{ Promastigotes } & \multicolumn{2}{|l|}{ Amastigotes } \\
\hline & & $\mathrm{EC}_{50-\mathrm{PRO}}$ & $\mathrm{SI}_{1}$ & $\mathrm{EC}_{50-\mathrm{AMA}}$ & $\mathrm{SI}_{2}$ \\
\hline $4 a$ & $152.2 \pm 2.2$ & $15.5 \pm 0.5$ & 9.8 & $>20.0$ & - \\
\hline $4 b$ & $180.1 \pm 25.8$ & $52.3 \pm 0.4$ & 3.4 & n.d. & - \\
\hline $8 \mathbf{a}$ & $63.2 \pm 0.6$ & $13.8 \pm 1.2$ & 4.6 & $18.2 \pm 0.1$ & 3.5 \\
\hline $8 b$ & $248.3 \pm 2.8$ & $42.8 \pm 3.3$ & 5.8 & $>20.0$ & - \\
\hline $8 c$ & $200.3 \pm 2.4$ & $21.9 \pm 2.4$ & 9.1 & $>20.0$ & - \\
\hline $14 a$ & $114.6 \pm 4.1$ & $47.9 \pm 2.9$ & 2.4 & n.d. & - \\
\hline $14 b$ & $32.1 \pm 5.8$ & $5.7 \pm 3.3$ & 5.6 & $3.1 \pm 0.2$ & 10.5 \\
\hline $14 c$ & $35.8 \pm 1.6$ & $6.2 \pm 2.4$ & 5.8 & $7.1 \pm 0.1$ & 5.0 \\
\hline $14 d$ & $45.6 \pm 1.5$ & $7.8 \pm 0.5$ & 5.9 & $2.1 \pm 0.1$ & 21.6 \\
\hline $14 \mathrm{e}$ & $208.3 \pm 0.2$ & $9.8 \pm 0.5$ & 21.3 & $3.1 \pm 0.1$ & 66.4 \\
\hline $14 \mathrm{f}$ & $23.5 \pm 2.2$ & $9.4 \pm 0.7$ & 2.5 & n.d. & - \\
\hline AmpB & $23.1 \pm 2.5$ & $0.9 \pm 0.1$ & 25.1 & $1.0 \pm 0.1$ & 23.3 \\
\hline
\end{tabular}

${ }^{\text {a }}$ (Dutra et al., 2014).

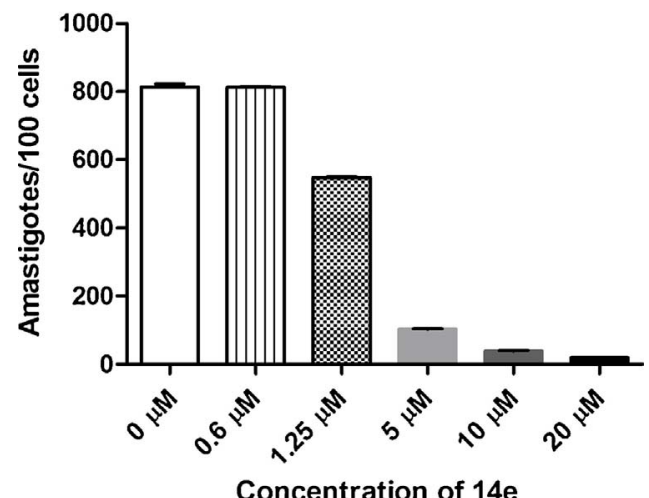

Fig. 1. In vitro phagocytosis of Leishmania infantum promastigotes by macrophages and treated with $14 \mathrm{e}$.

Cells were infected with $L$. infantum promastigotes (at a 1:10 cell to parasite ratio). The cells were treated with 14e for $24 \mathrm{~h}$. The cells were stained with Giemsa, and the phagocytic index was calculated as described in the Materials and Methods. Bars denote the average of three different experiments.

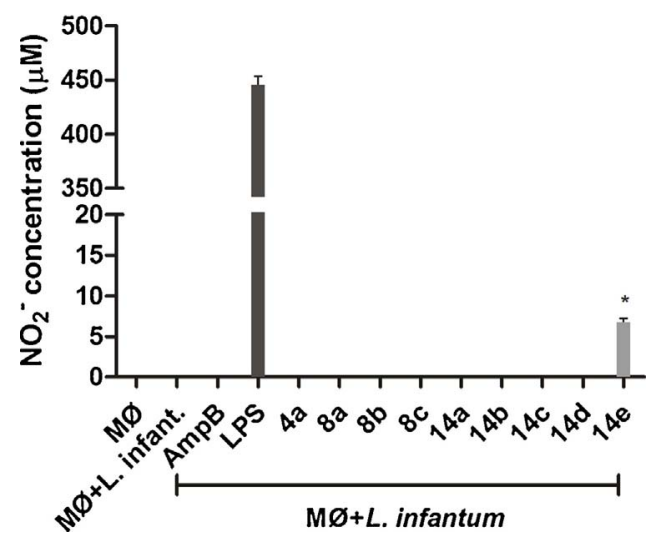

Fig. 2. Nitric oxide production in supernatant of Leishmania-infected cells treated with furoxan and benzofuroxan derivatives.

NO production by Leishmania-infected macrophages treated with LPS, amphotericin B (AmpB), and compounds 4a, 8a-8c, and 14a-14e at $10.0 \mu \mathrm{g} / \mathrm{mL}$. As controls were used untreated Leishmania-infected macrophages and non-infected macrophages. The data are expressed as average \pm SD.*: Statistical significance of the difference relative to the positive control (treatment with LPS) $(P<0.05)$ was determined by Tukey's test.

$\left(\mathrm{EC}_{50-\mathrm{PRO}}=47.9 \mu \mathrm{M}\right)$ nor a favorable selectivity index $\left(\mathrm{SI}_{1}=2.4\right)$. The benzofuroxan derivatives $(\mathbf{8} \mathbf{a}-\mathbf{c})$ did not present outstanding results; among them, despite the fact that derivative $8 \mathbf{a}\left(\mathrm{EC}_{50}=13.8 \mu \mathrm{M}\right.$,
(A)

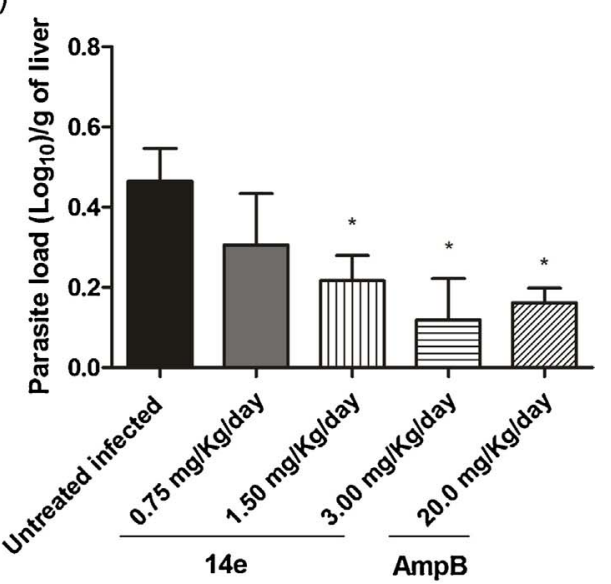

(B)

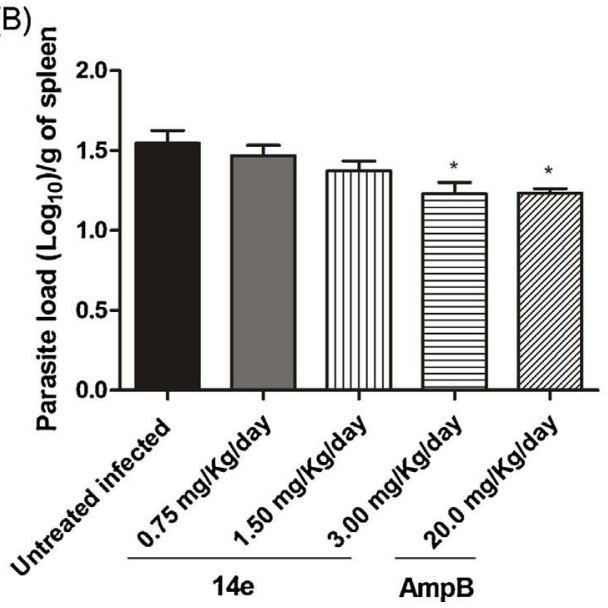

Fig. 3. Treatment of L. infantum-infected hamsters with $14 \mathrm{e}$ or AmpB.

Groups of five hamsters were infected with $L$. infantum promastigotes in stationary phase, followed 75 days post-infection by treatment of the animals with $14 \mathrm{e}$ or AmpB. The parasite load was determined by the limiting dilution method at the end of the treatment in (A) in the liver and (B) in the spleen. The data are expressed as average \pm SD.*: Statistical significance of the difference relative to the untreated infected group $(\mathrm{P}<0.05)$ was determined by Tukey's test.

$\left.\mathrm{SI}_{1}=4.6\right)$ has shown good anti-parasitic activity, the cytotoxicity studies demonstrated its low selectivity to the parasite versus the macrophages. From this group of molecules, compounds $4 a, 8 a-c$, and 14b$14 \mathrm{e}$ that presented $\mathrm{SI}_{1}$ values larger than or equal to five were then selected for determination of antileishmanial activity against intracellular amastigotes, the clinically relevant form of Leishmania species.

\subsection{Efficacy of furoxan and benzofuroxan derivatives against L. infantum- Infected macrophages}

L. infantum-infected macrophages were exposed to compounds $4 a$, 8a-c, and 14b-14e at different concentrations ( 0.6 to $20 \mu \mathrm{M})$ for a $24 \mathrm{~h}$ period (Table 2). Compounds $14 \mathbf{b}-\mathbf{1 4} \mathbf{e}$ were active against intracellular amastigotes. Compounds $14 \mathrm{~b}\left(\mathrm{EC}_{50-\mathrm{AMA}}=3.1 \mu \mathrm{M}, \mathrm{SI}_{2}=10.5\right)$, $-\mathrm{d}$ $\left(\mathrm{EC}_{50-\mathrm{AMA}}=2.1 \mu \mathrm{M}, \quad \mathrm{SI}_{2}=21.6\right)$ and $-\mathrm{e} \quad\left(\mathrm{EC}_{50-\mathrm{AMA}}=3.1 \mu \mathrm{M}\right.$, $\mathrm{SI}_{2}=66.4$ ) presented 2 to 10 -times lower anti-amastigote activity than AmpB $\left(\mathrm{EC}_{50 \mathrm{AMA}}=1 \mu \mathrm{M}, \mathrm{SI}_{2}=23.3\right)$. However, compound $14 \mathrm{e}$ was about 3-times less cytotoxic to the mammalian cells compared to the reference drug. Moreover, this compound showed a dose-dependent curve against the intracellular amastigotes (Fig. 1). 
(A)

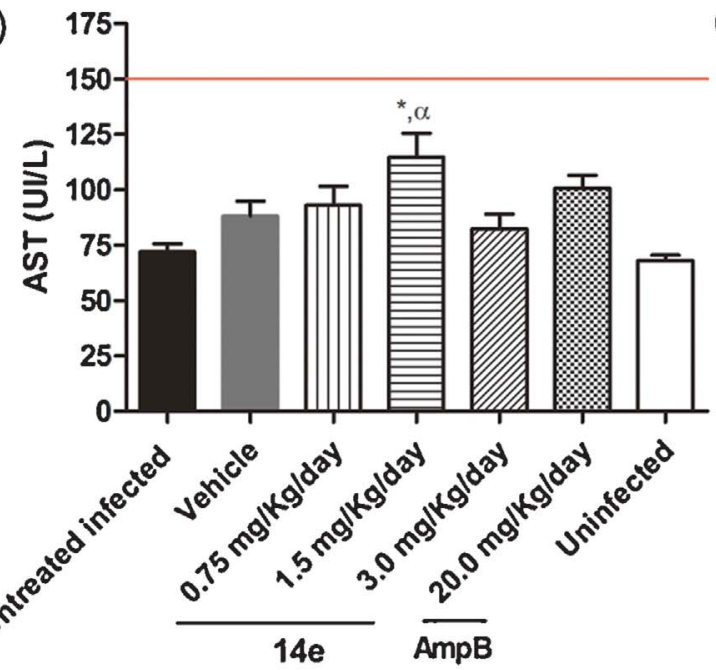

(C)
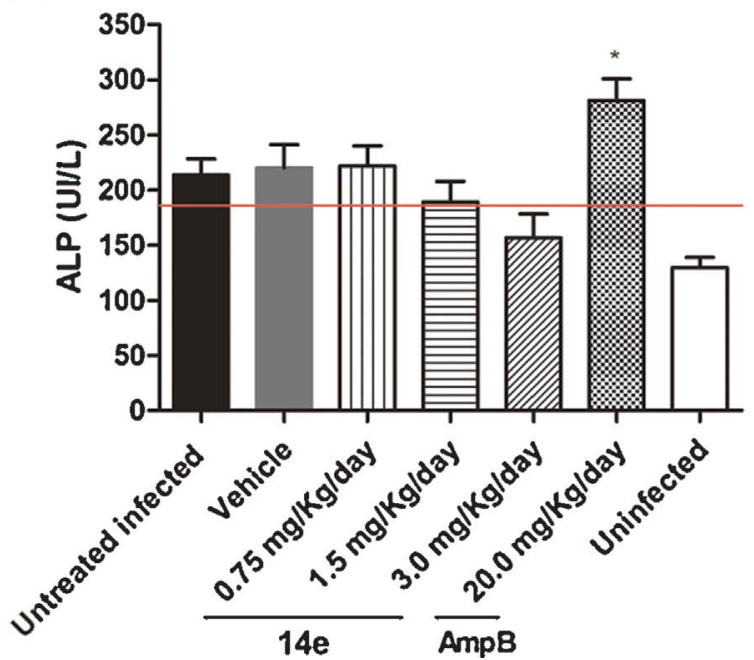

(B)

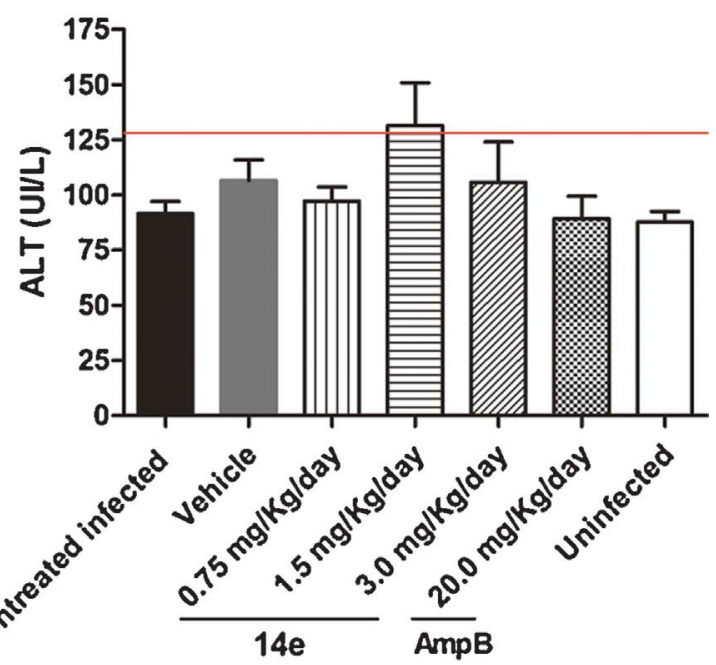

(D)

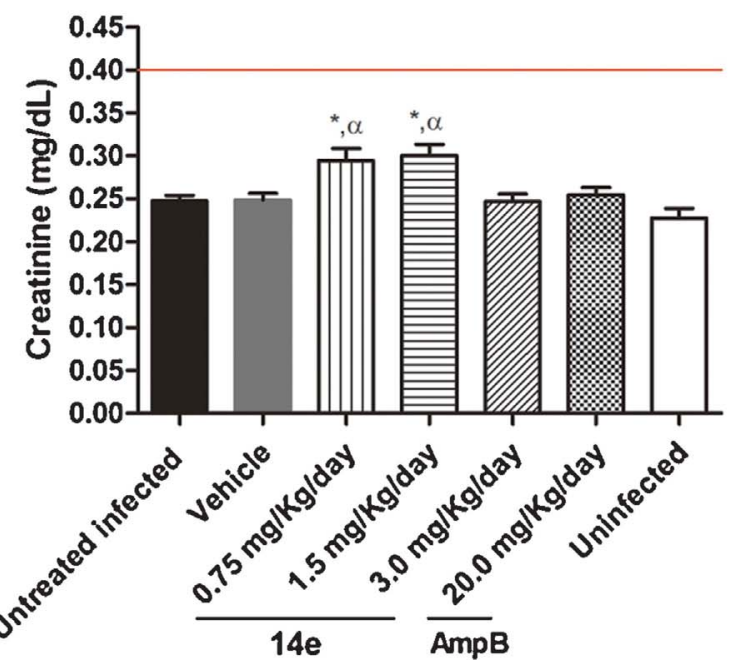

Fig. 4. Toxicity evaluation in $L$. infantum-infected hamster after treatment with $14 \mathrm{e}$ or AmpB.

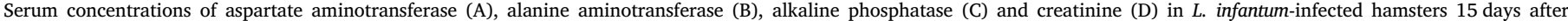

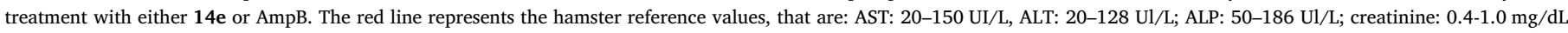

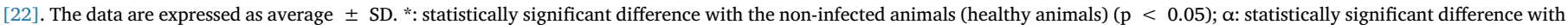
untreated infected animals $(\mathrm{p}<0.05)$.

\subsection{In vitro nitric oxide production}

Culture supernatants from murine $L$. infantum-infected peritoneal macrophages exposed to $10 \mu \mathrm{g} / \mathrm{mL}$ of the compounds $4 \mathbf{a}, \mathbf{8 a}-\mathbf{8 c}$, and 14b-14e and LPS (positive control) were evaluated for indirect NO production. Among all evaluated furoxans, compound 14e was the only one capable of increasing the levels of NO to $7 \mu \mathrm{M}$ in the cells supernatants, although to a lesser extent than LPS (0.45 mM) (Fig. 2).

\subsection{Efficacy of $14 e$ on golden hamster infected with $L$. infantum}

Golden hamsters infected with $L$. infantum were treated (10 weeks post infection) for 15 days with three different doses of $14 \mathrm{e}(3,1.5$ or $0.75 \mathrm{mg} / \mathrm{Kg} /$ day). For the same period, the control group was treated with AmpB (20 mg/Kg/day). The animals treated with both $14 \mathrm{e}$ and AmpB displayed reduction in the parasite burden. Briefly, 14e decreased the number of parasites in a dose-dependent manner $(3,1.5$ or $0.75 \mathrm{mg} / \mathrm{Kg} /$ day) in both liver $(54.2 \%, 43.7 \%$ and $13.5 \%)$ and spleen (49.9\%, 33.2\% and $16.9 \%)$. Although the reduction of parasite burden in the liver (50.5\%) and spleen (51.5\%) of $L$. infantum-infected hamsters treated with AmpB was similar to those obtained in the $14 \mathrm{e}$ treatment, it was necessary to use an AmpB dose seven times higher than that of 14e to achieve similar effect (Fig. 3A and B).

\subsection{Evaluation of biomarkers of hepatic and renal function}

In order to investigate if $14 \mathrm{e}$ treatment might cause hepato- and/or nephrotoxicity in the treated hamsters, possible changes in the liver and kidney function were monitored by measuring the serum levels of AST, ALT, ALP and creatinine. For the liver function, no significant alterations were observed in the levels of both ALT and AST for any of the analysed groups of animals. However, the levels of ALP were above the reference values for both treated animals with $14 \mathrm{e}(0.75$ and $1.5 \mathrm{mgKg}$ / day) and AmpB. Moreover, only AmpB showed statistically significant differences with the non-infected group with regard to the ALP levels (Fig. 4C). Serum levels of creatinine in all groups were below the reference values [22] (Fig. 4D). Taken all together, these data indicate that $14 \mathrm{e}(3.0 \mathrm{mg} / \mathrm{Kg} /$ day $)$ is safer than AmpB.

\subsection{Histopathological analysis}

Histopathological analyses of liver, spleen and kidney were carried 
(A)

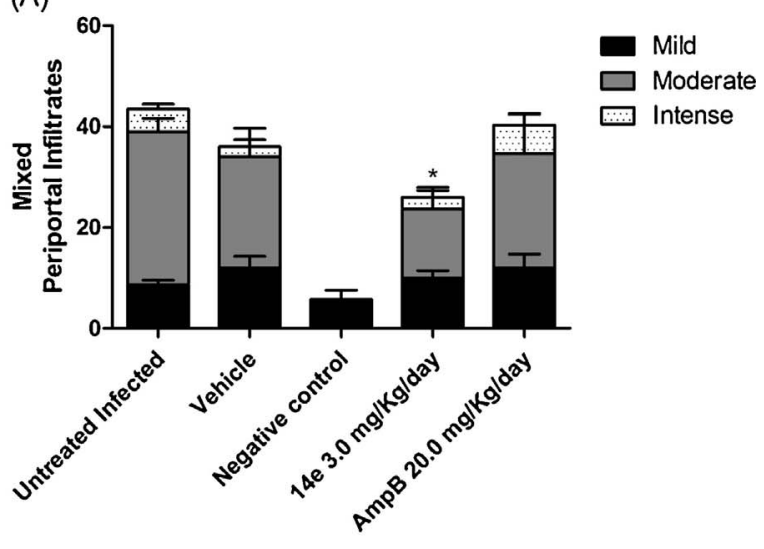

(B)

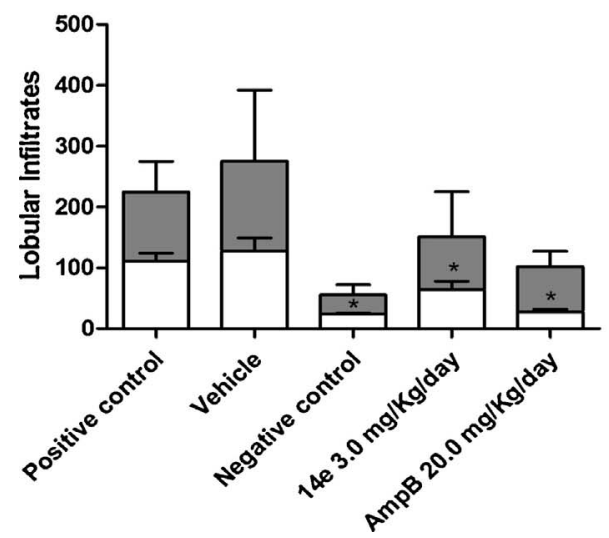

Fig. 5. Inflammatory infiltrates in the liver of $L$. infantum-infected hamster after treatment.

Groups of five hamsters were infected with $L$. infantum promastigotes in stationary phase, followed 75 days post-infection by treatment of the animals with $\mathbf{1 4} \mathrm{e}$ or AmpB. Liver sections were paraffin sectioned and stained with $\mathrm{H} \& \mathrm{E}$, and analyzed under a microscope. (A) Mixed periportal infiltrates in tested groups, (B) type of lobular infiltrates. The data are expressed as average \pm SD.*: Statistical significance of the difference relative to the untreated infected group $(\mathrm{P}<0.05)$ was determined by Tukey's test.

out in all experimental groups by analyzing, for each animal, the entire glass slide containing the sample. All analyses were blindly performed by a pathologist, who did not know the experimental groups.

For the liver, no statistical diferences were observed between animals of the untreated infected group and those infected and treated with the vehicle. The periportal region showed high frequency of inflammatory infiltrates, which were predominantly mixed and moderate. In addition, these groups also showed a significant amount of large lobular inflammatory infiltrates of both types: mixed and chronic (Figs. 5 A, B, 6 A and B). For both groups, the presence of amastigotes in liver tissue was observed (Fig. 7A). The group treated with compound $14 \mathrm{e}$ at $3 \mathrm{mg} / \mathrm{Kg} /$ day showed a reduced number of mixed inflammatory infiltrates (moderate and intense) in the periportal region compared to untreated infected control animals (Figs. $5 \mathrm{~A}$ and $6 \mathrm{D}$ ). Interestingly, the lobular region also presented a reduced number of mixed inflammatory infiltrates (Fig. 5B). Moreover, empty vacuoles were seen in this group (Fig. 7B). Interestingly, animals treated with the other two lower doses of compound $14 \mathrm{e}(0.75$ and $1.5 \mathrm{mg} / \mathrm{Kg} /$ day $)$ as well as those treated with AmpB presented similar number of periportal and lobular inflammatory infiltrates to that seen in the untreated infected animals (Figs. 5 A, 6 E-G). For the AmpB group, the lobular region showed a reduction in the number of mixed infiltrates comparable with that observed when the highest dose of compound $14 \mathrm{e}$ was administered (Fig. 5B).

In this study, the spleen of all groups showed an intact capsule, but some diferences were observed in the white and red pulp, marginal zone and follicules. In the untreated infected group was seen that the Leishmania infection caused some alterations in the spleen, such as hypoplasia of white pulp accompanied by loss of structural definition, followed by an increase of the marginal zone (Fig. 8A) as well as presence of granulomas and amastigotes (Fig. 8B). These same findings were made in the group of infected animals treated with the vehicle (Fig. 8B); no statistically significant differences were found. Futhermore, the non-infected group possessed normal white pulp and marginal zone, as expected (Fig. 8C). The spleen of infected animals treated with the highest dose of $14 \mathrm{e}(3 \mathrm{mg} / \mathrm{Kg} /$ day $)$ revelead a normal proportion between white/red pulp, although the marginal zone was unexpectedly increased (Fig. 8D). Moreover, parasites were rarely found (Fig. 7D). The lowest doses of compound $14 \mathrm{e}(0.75$ and $1.5 \mathrm{mg} / \mathrm{Kg} /$ day) resulted in hypoplasia of white pulp, increased marginal zone (Fig. 8E and F) and identification of amastigotes in the spleen, as observed for the untreated infected group. The treatment with AmpB at $20 \mathrm{mg} / \mathrm{Kg}$ /day showed severe hypoplasia of white pulp, and in some cases this was corroborated by loss of structural definition (Fig. 8G); in this group parasites were also rarely found. AmpB treatment induced a thickening of the capsule and subcapsular abscess (Fig. 8H), which was not seen in other groups.

Analysis of the kidney revealed that all groups exhibited intact Bowman's capsule; the proximal and distal ducts in cortical region were normal in the majority of the animals with no statistical differences between each group. However, differences were observed in the Bowman's capsule space and in the ducts of the medular area. In the untreated infected animals, a reduction in the Bowman's capsule space was observed (Fig. 9A). For this group a granuloma was observed in the cortical area and edema in the ducts of the medular area. Similar alterations in the kidney were verified in the infected and vehicle-treated group (Fig. 9B), which however did not show statistically significant differences compared to the untreated infected animals. Treatment with compound $14 \mathrm{e}$ ( 3 and $1.5 \mathrm{mg} / \mathrm{Kg} /$ day) revelead normal Bowman's capsule space and ducts, showing that this compound did not induce toxicity in this organ (Fig. 9D and E). Compound 14e at $0.75 \mathrm{mg} / \mathrm{Kg} /$ day exhibited the same pattern as seen in the untreated infected group (Fig. 9F). Damage had occurred in the kidney of the AmpB group which showed a reduction in the Bowman's capsule space (Fig. 9G), accompanied by edema in the proximal ducts as well as necrotic and picnotic areas (Fig. 9H) that suggest a nephrotoxic effect induced by AmpB.

\section{Discussion}

VL is the most serious life-threatening form of leishmaniasis, one of the six major diseases targeted by WHO. No vaccine is yet available for human use and chemotherapy is the only way of dealing with this disease [23]. However, the few chemotherapeutical options associated with long-term treatment and high toxicity that cause often abandonment of the treatment and, consequently, the appearance of resistant strains justify the joint efforts of the scientific community, non-profit research institutions and pharmaceutical companies to discover new drugs against this extremely neglected disease [24].

$\mathrm{N}$-oxide compounds including furoxan and benzofuroxan have been shown as promising scaffolds for designing of antiparasitic drugs. Indeed, these derivatives have been explored as pharmacophores for Schistosoma, Plasmodium and Leishmania [13-16,25-27]. We have previously demonstrated the synthesis, characterization and evaluation of furoxan and benzofuroxan derivatives against promastigote and amastigote forms of L. amazonensis [17]. Herein we evaluated both the in vitro and in vivo efficacy of $14 \mathrm{e}$ in $L$. infantum parasites elimination.

The leishmanicidal activity as observed for compound 14e against both promastigotes $\left(\mathrm{EC}_{50-\mathrm{PRO}}=9.8 \mu \mathrm{M} ; \mathrm{SI}_{1}=21.3\right)$ and amastigotes $\left(\mathrm{EC}_{50-\mathrm{AMA}}=3.1 \mu \mathrm{M} ; \mathrm{SI}_{2}=66.4\right)$ seems promising. It is worth to mention that $14 \mathrm{e}$ exhibited the highest SI values for both parasite forms $\left(\mathrm{SI}_{1}=21.3\right.$ and $\left.\mathrm{SI}_{2}=66.4\right)$, which is about three times more selective 
(A)

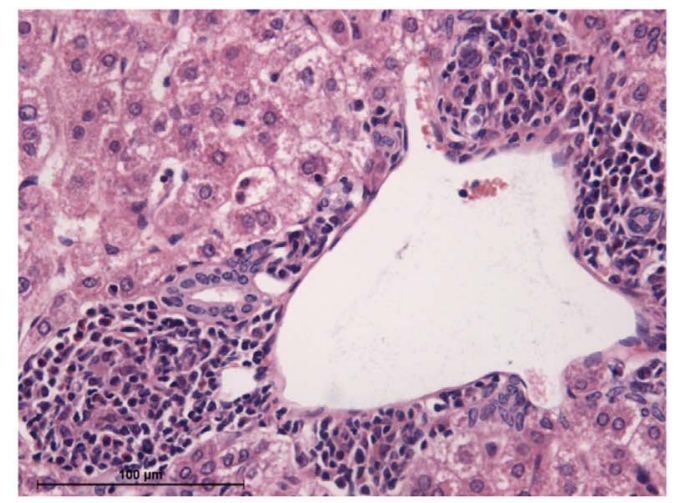

(C)

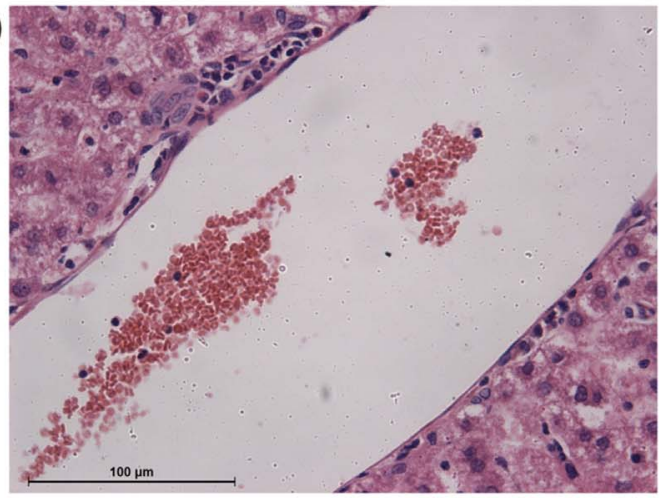

(E)

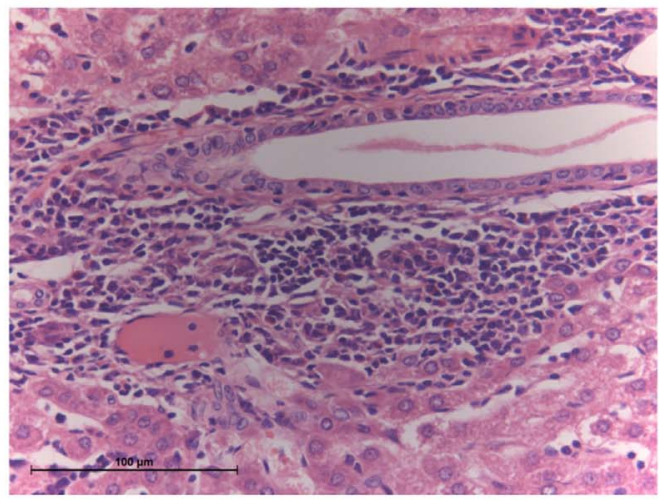

(G)

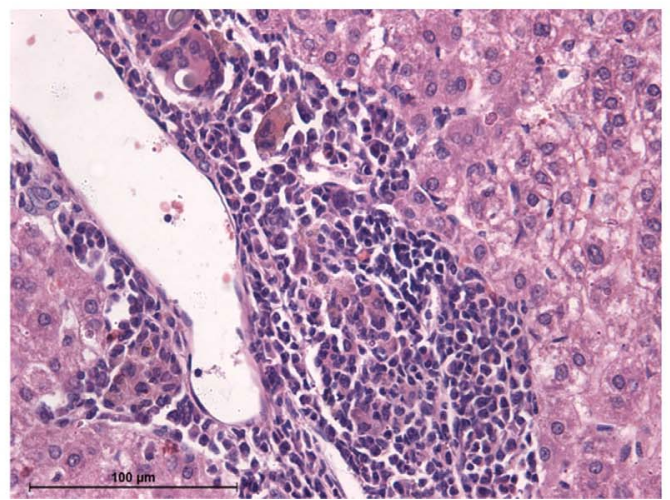

(B)

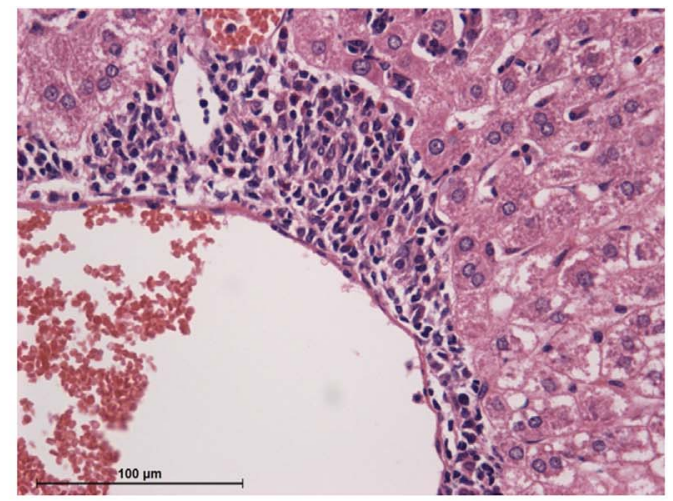

(D)

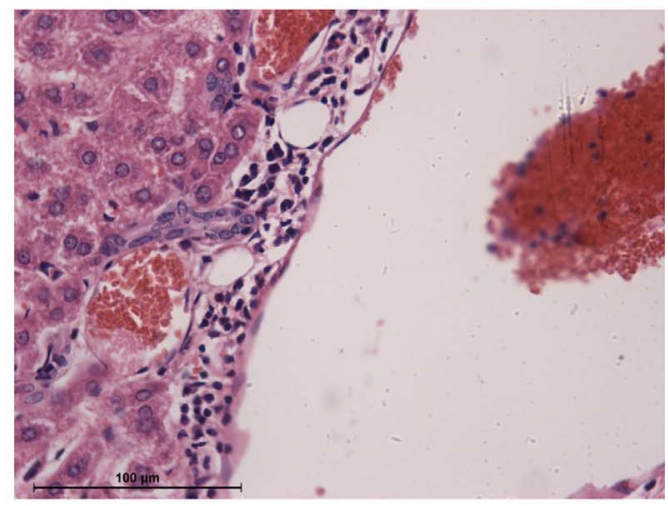

(F)

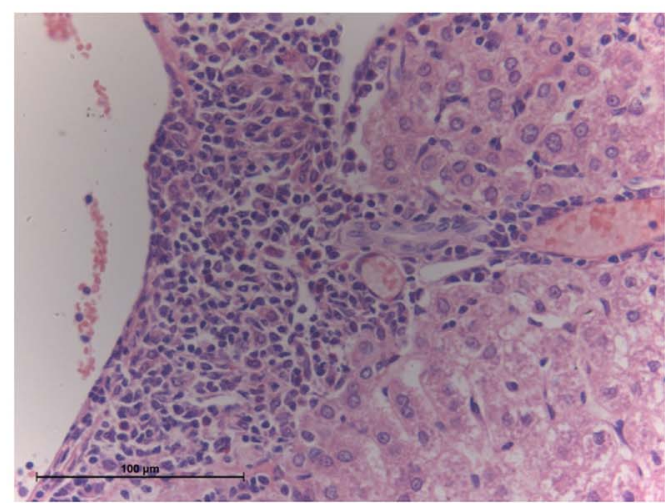

(H)

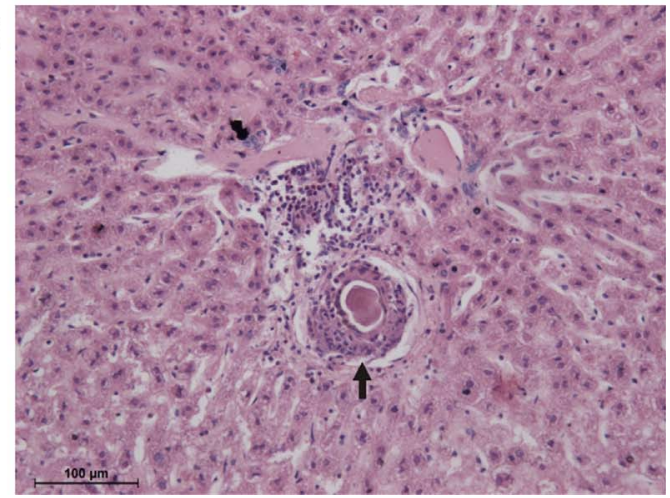

Fig. 6. Histopathological alterations in the liver of $L$. infantum-infected hamster after treatment.

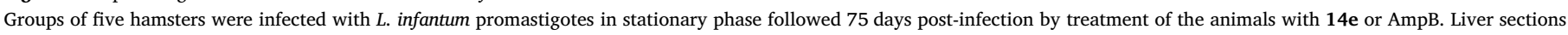

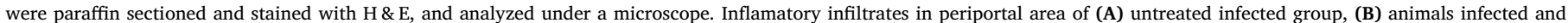

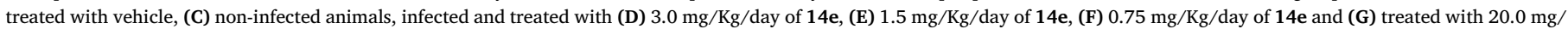
$\mathrm{Kg}$ /day of AmpB. $40 \mathrm{x}$ magnification. In (H) focus in a Schaumann' bodies in the untreated infected group (20 x magnification). 

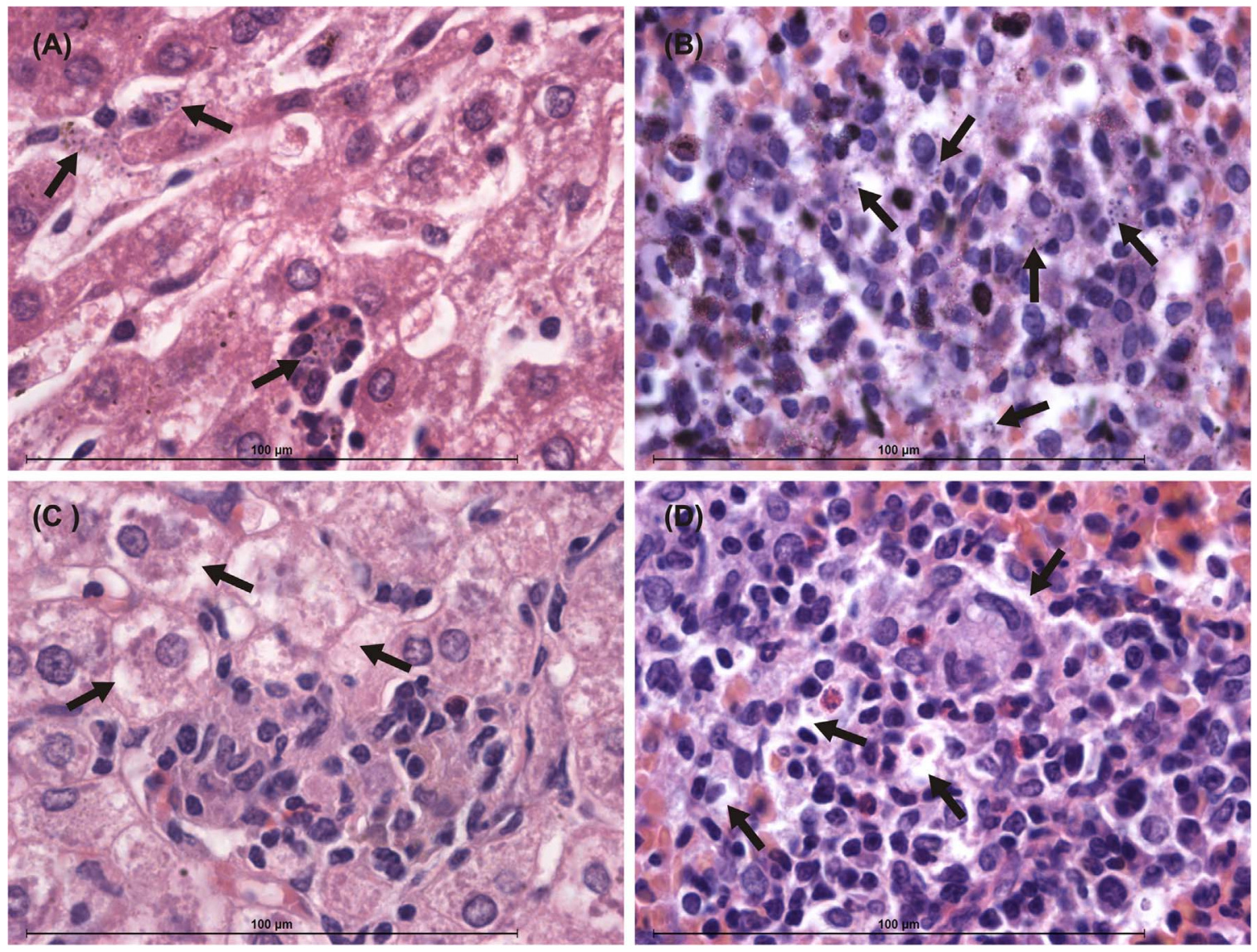

Fig. 7. Amastigotes in the liver and spleen of $L$. infantum-infected hamster after treatment.

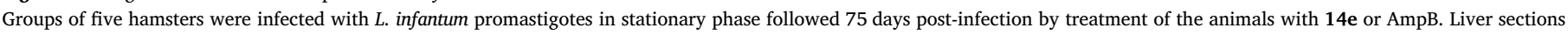

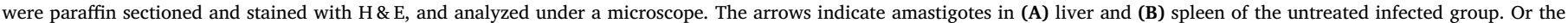
arrows indicate empty vacuoles in (C) liver and (D) spleen of animals treated with $3.0 \mathrm{mg} / \mathrm{Kg} / \mathrm{day}$ of $14 \mathrm{e} .100 \mathrm{x}$ magnification.

to the amastigotes, the clinically relevant form of Leishmania species, than the reference drug AmpB. We also observed that $14 \mathrm{e}$ was able to reduce the number of infected macrophages as well as the number of intracellular amastigotes, which demonstrate its effectiveness in parasite clearance.

VL is fatal if left untreated and is caused by the intracellular parasitic protists L. donovani or L. infantum (chagasi) [33]. Syrian golden hamsters (Mesocricetus auratus) have been described as a useful model of many human infectious diseases since they exhibit susceptibility to a variety of intracellular pathogens, such as different species of Leishmania, including $L$. infantum [34-39]; besides, this model has been used for the in vivo tests of new antileishmanial compounds [40,41]. The efficiency of compound $14 \mathrm{e}$ to eliminate the parasite in L. infantuminfected hamsters was also demonstrated. $14 \mathrm{e}$ led to a significant reduction of parasite load in animal livers $(54.2 \%, 43.7 \%$ and $13.5 \%)$ and spleens $(49.9 \%, 33.2 \%$ and $16.9 \%)$ in a dose-dependent manner $(3,1.5$ and $0.75 \mathrm{mg} / \mathrm{Kg} /$ day).

Moreover, $14 \mathrm{e}$ at $3.0 \mathrm{mg} / \mathrm{Kg} /$ day did not cause toxicity in $L$. infantum-infected hamsters as demonstrated by levels of the hepatic transaminases (ALT/AST) and the renal biomarker (creatine) assayed after drug treatment when compared to the reported references values [22]. On the other hand, high levels of ALP were found in the untreated infected group and infected animals treated with AmpB and the lower doses of $14 \mathrm{e}(1.5$ and $0.75 \mathrm{mg} / \mathrm{Kg} / \mathrm{day})$. In fact, the observed hepatic changes after AmpB treatment might be partly due to the toxic effect of this drug since some authors have described elevated levels of ALP after AmpB treatment [42]. It is interesting to note that ALP levels are inversely proportional to the increased dose concentration of $14 \mathrm{e}$ and might be attributed to the parasite clearance in this organ.
In order to check possible histopathological alterations, samples from the liver, the spleen and the kidney were taken from animals of all groups, paraffin sectioned and stained with $\mathrm{H} \& \mathrm{E}$. The inflammatory process in the liver containing VL involves parasitised macrophages and lymphocytes, and this process affects the portal tracts and is found also inside the lobule [43]. Moreover, alterations have been described in hepatic tissue in animals with VL characterized by the presence of inflammatory infiltrates [44,45]. Indeed, a high frequency of inflammatory infiltrates (predominantly mixed and moderate) was observed in periportal tracts of the untreated infected group and infected animals treated with vehicle (Fig. 7A and B). We have also identified amastigotes in the liver tissues (Fig. 8A). Treatment with compound $14 \mathrm{e}$ at $3 \mathrm{mg} / \mathrm{Kg} /$ day resulted in a reduction of $54.9 \%$ and $48.9 \%$ in respectively moderate and intense inflammatory infiltrates in periportal tracts compared to the untreated infected group. In the lobular region, there was also a reduction in the number of mixed inflammatory infiltrates. Schaumann's bodies have been associated with a deficient phagocytic macrophage system and have a strong association with Leishmania infection [46,47], since Schaumann's bodies originate from residual bodies that are end-products of activated lysosomes [48]. Indeed, Schaumann's bodies were observed in the untreated infected group as can be seen in the (Fig. $7 \mathrm{H})$. On the other hand, the treatment with compound $14 \mathrm{e}$ at the highest dose $(3 \mathrm{mg} / \mathrm{Kg} /$ day $)$ reduced the number of Schaumann's bodies compared to those in the untreated infected group, suggesting a recovery of the phagocytic macrophage system.

The murine spleen is a highly organized lymphoid organ consisting of red and white pulp, which is composed of three subcompartments: the periarteriolar lymphoid sheath, the follicles, and the marginal zone. 
(A)

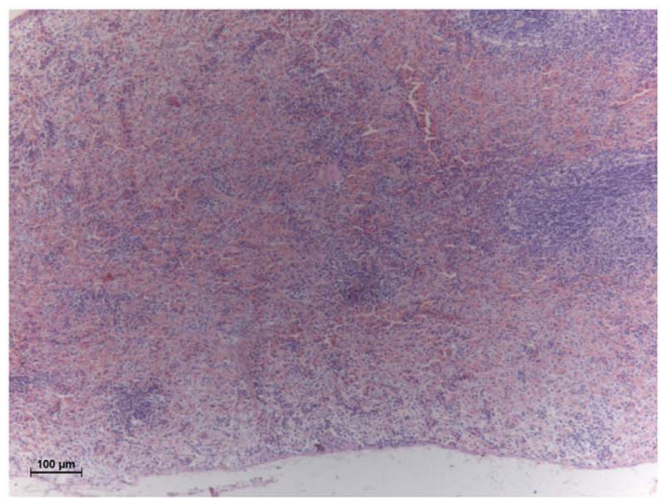

(C)

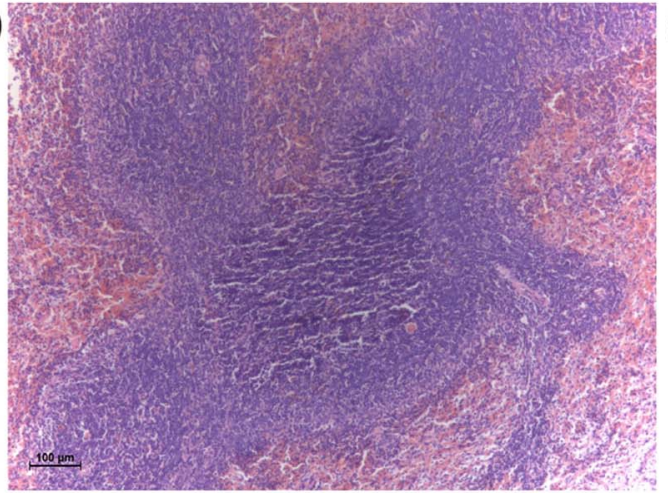

(E)

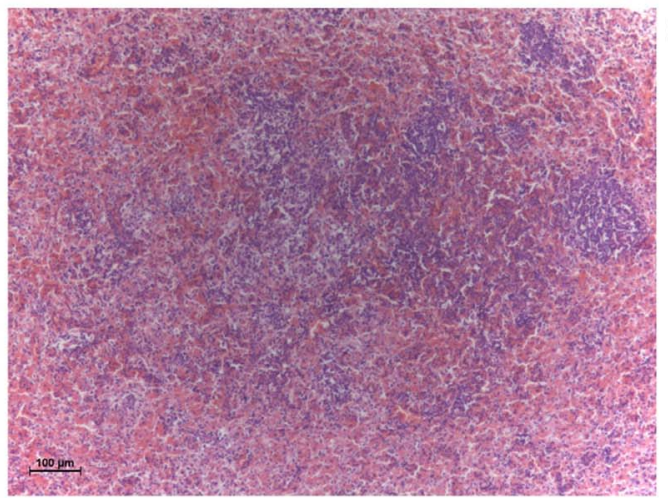

(G)

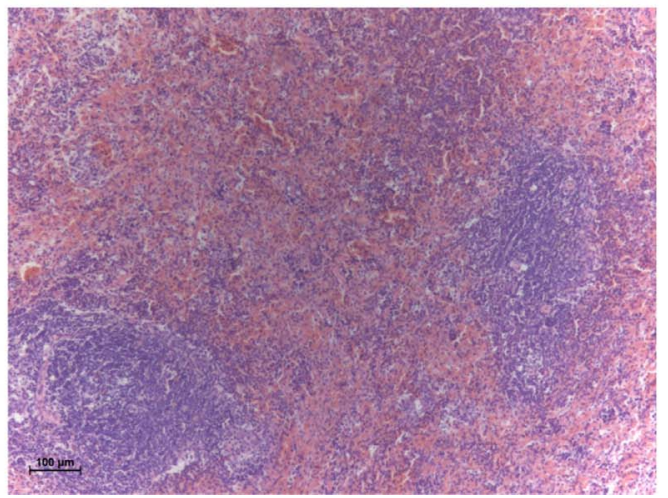

(B)

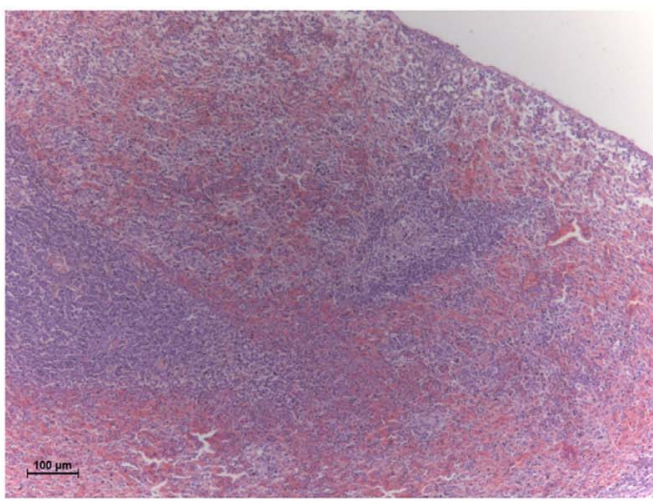

(D)

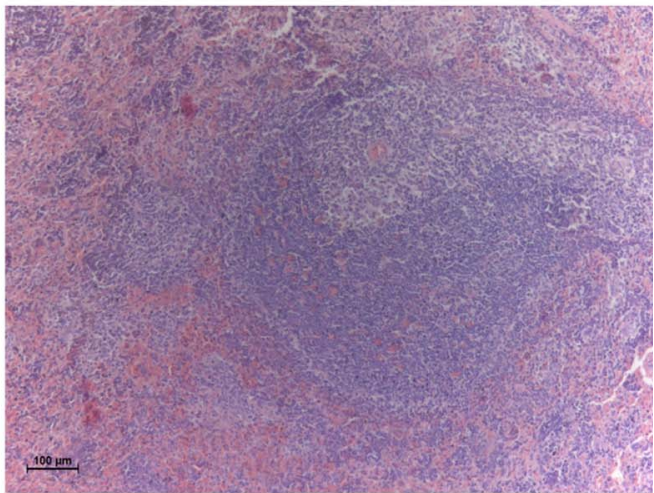

(F)

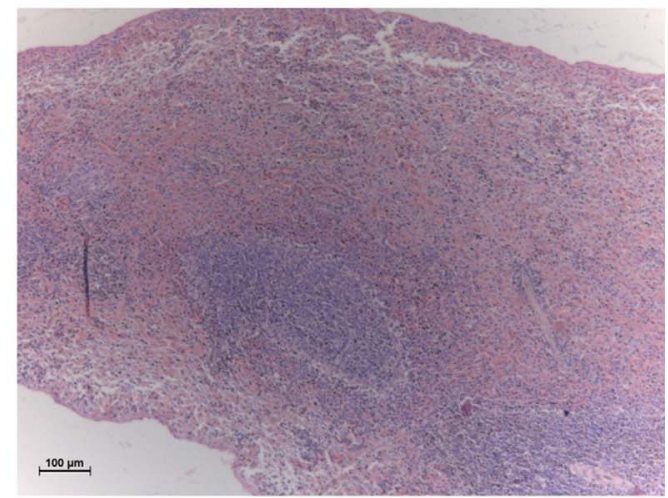

(H)

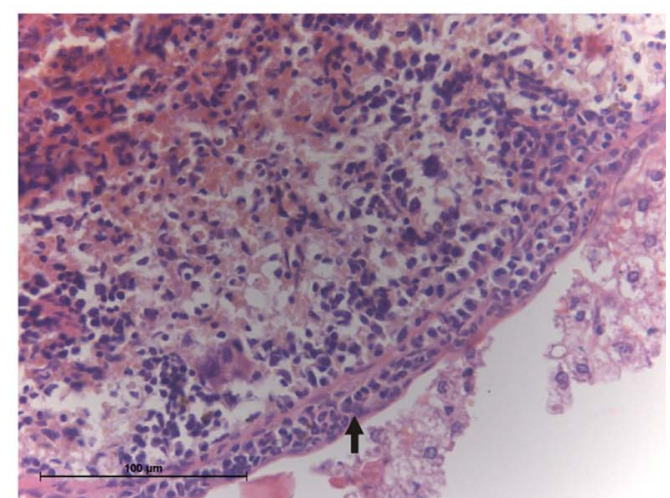

Fig. 8. Histopathological alterations in the spleen of $L$. infantum-infected hamsters after treatment.

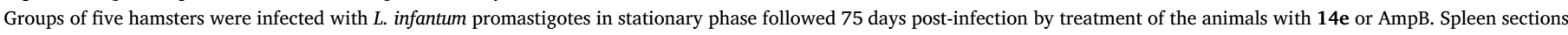

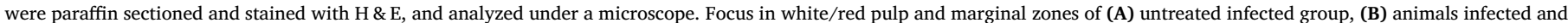

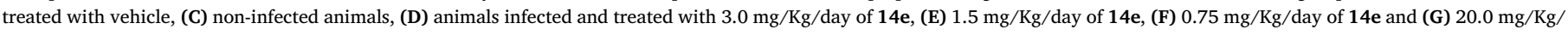
day of AmpB. $10 \mathrm{x}$ magnification. In (H) focus in a subcapsular abscess in the infected and treated with AmpB group (40 x magnification). 
(A)

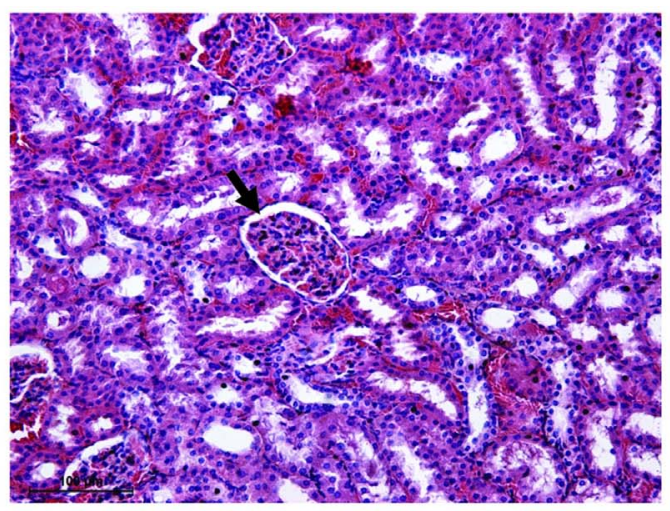

(C)

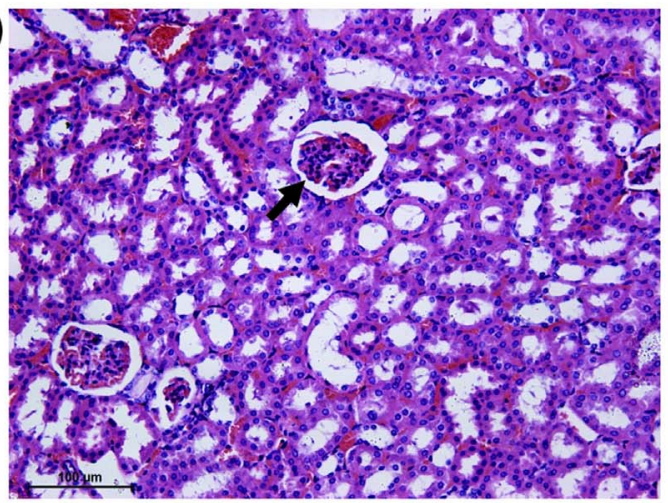

(E)

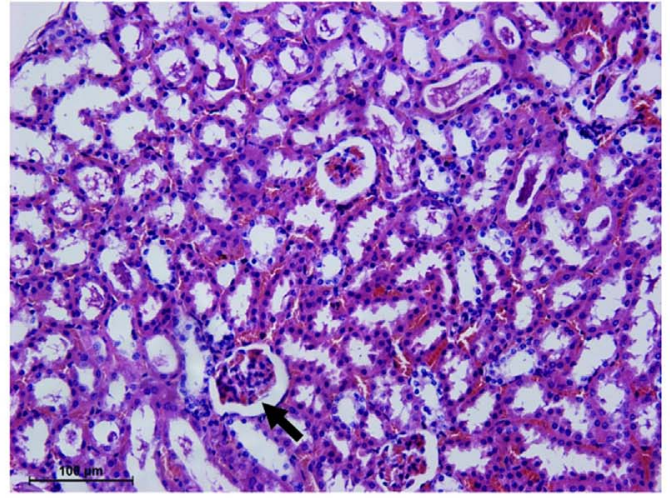

(G)

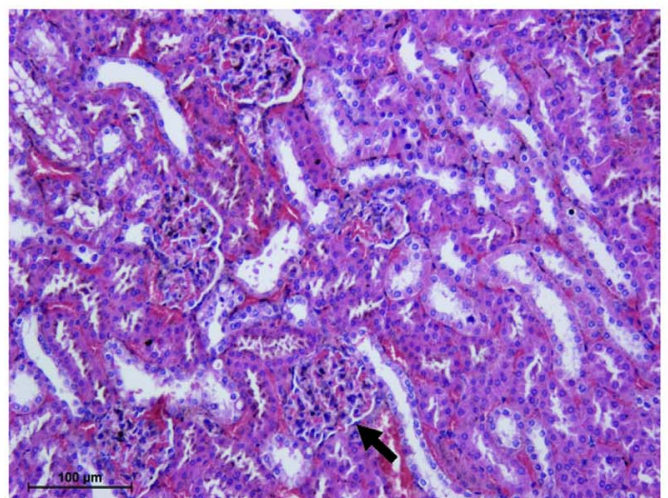

(B)

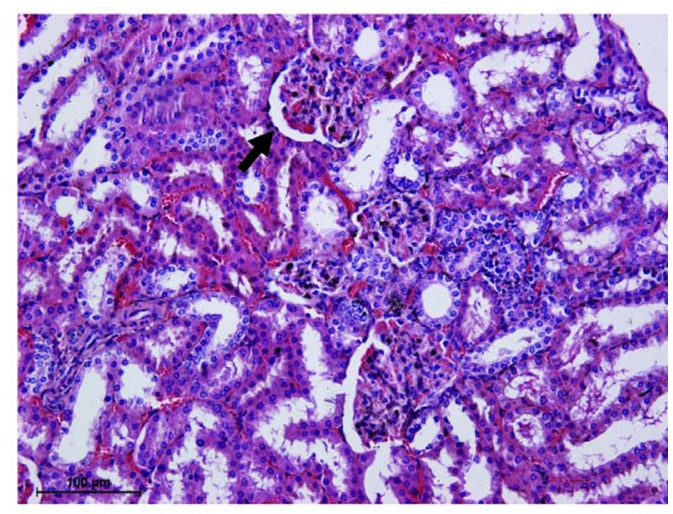

(D)

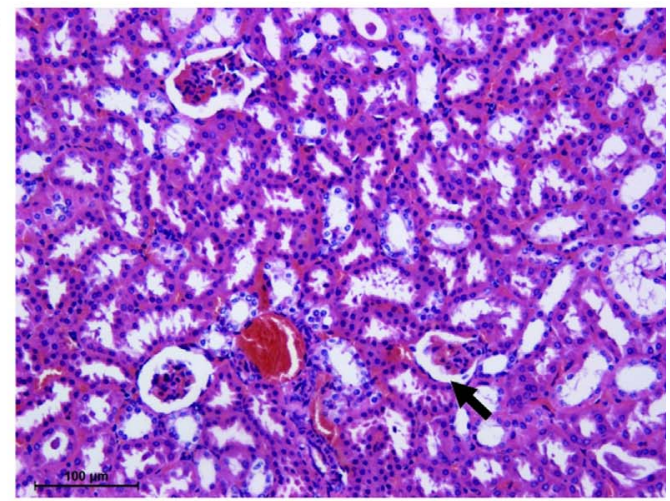

(F)

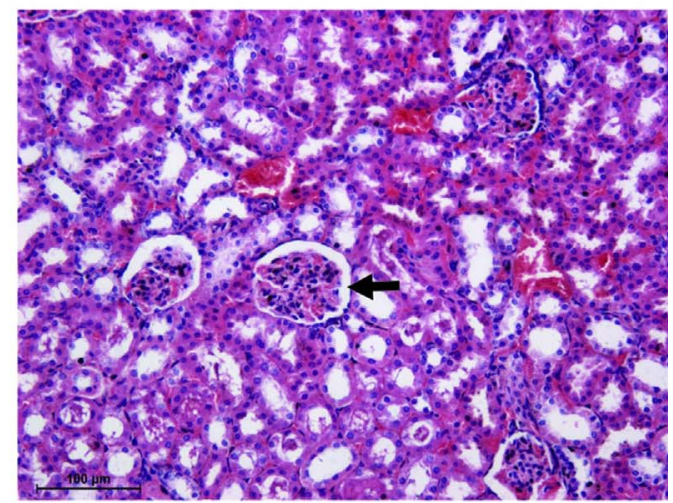

(H)

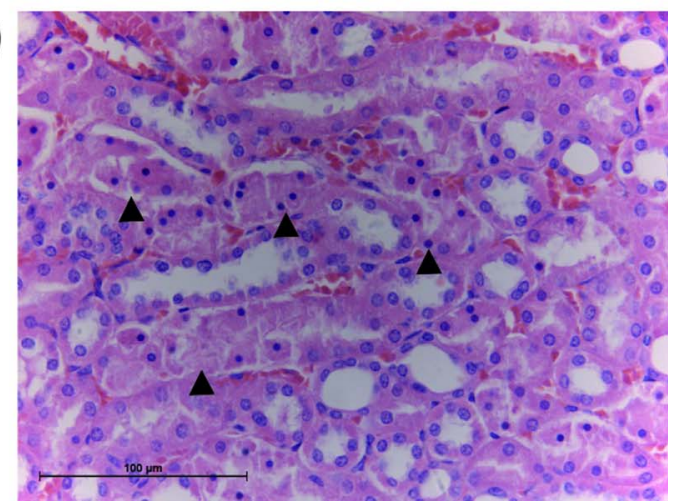

Fig. 9. Histopathological alterations in the kidney of $L$. infantum-infected hamster after treatment.

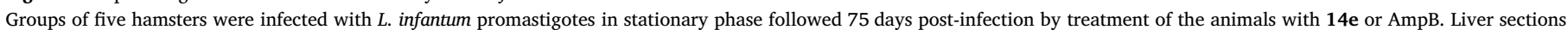

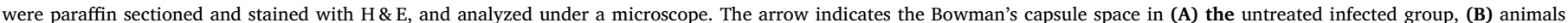

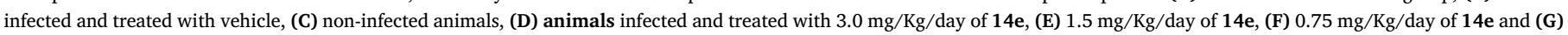
$20.0 \mathrm{mg} / \mathrm{Kg} /$ day of AmpB. $20 \mathrm{x}$ magnification. In (H) the arrowhead indicate necrotic/picnotic areas in the infected and treated with AmpB group (40 x magnification). 
Some authors have described an early invasion of this organ after the establishment of a visceral infection [49]. Moreover, many pathological changes associated with human VL are observed in the spleen. During chronic stages of infection ( $>28$ days), an immune dysfunction is associated with a disorganization of white pulp such as size reduction. Moreover, it is possible to observe heavily infected macrophages in multiple and unusual locations [50]. In our study, the infection of syrian Golden hamsters by $L$. infantum caused some alterations in the spleen as described above, including white pulp hypoplasia accompanied by an increase in the marginal zone (Fig. 8A). Histological analyses also revelead granuloma formation and presence of parasites in the spleen of untreated infected animals (Fig. 8B). The group treated with AmpB (20.0 mg/Kg/day) exhibited severe hypoplasia of white pulp followed by loss of a defined organ structure (Fig. 8G) and the appearance of granuloma; however, parasites were rarely found. The AmpB group also presented fibrous thickening of the splenic capsule accompanied by a subcapsular abcess (Fig. 8H), which was not observed in other groups. The marginal zone hyperplasia can occur with a variable degree of follicle atrophy, when associated with the obliteration of the boundaries between distinct areas of the lymphoid tissue, indicative of a structural disorganization of the white pulp. This result is in accordance with the observations described by Veress et al. [51]. Futhermore, the treatment with compound $14 \mathrm{e}$ (at $3 \mathrm{mg} / \mathrm{Kg} /$ day) revelead a normal relation between white/red pulp, within an expansion of marginal zone (Fig. 8D). It is worth to mention the importance of marginal zone, when associate with normal white pulp, in the immune response generation against pathogens $[52,53]$. This could indicate a recovery of the immune system after $14 \mathrm{e}$ treatment. The presence of granuloma was also observed; however, parasites were rarely found (Fig. 7D).

In order to confirm the absence of toxicity by $14 \mathrm{e}$ to the renal function, we have performed a histopathological analysis of the kidney to evaluate possible tissue damage. The analyses of Bowman's capsule space were made to examine a possible correlation between glomerulus response to toxic agents and reduction in the Bowman's capsule space. For the untreated infected group, a reduction in the Bowman's capsule space (Fig. 9A), the presence of granuloma in the cortical region and edema in the ducts of the medular area were verified. Despite the fact that we did not observe most of the such alterations in the group of animals treated with compound $14 \mathrm{e}$ at 1.5 or $3 \mathrm{mg} / \mathrm{Kg} /$ day, three out of five animals of this group exhibited edema in the ducts of the medular area. However, it is worth to emphasize that we did not observe any reduction in the Bowman's capsule space after $14 \mathrm{e}$ treatment at the dose of $3 \mathrm{mg} / \mathrm{Kg} /$ day (Fig. 9D). On the other hand, the reference drug AmpB induced kidney damage by reduction of the Bowman's capsule space accompanied by necrotic and picnotic areas (Fig. 9G and H); in addition, three out of five animals of this group also exhibited edema in ducts of the medular area. Contrary to the result obtained using creatinine as a biochemical marker for renal damage, these findings confirm the nephrotoxic effect of AmpB previously reported in the literature [54-60]. Indeed, relying on measuring creatinine might lead to delays in renal damage diagnosis because of its slow increase in serum after organ injury [61-63]. Thus, our data suggest that the combination of biochemical and histopathological analyses provide more accurate data about drug toxicity at the pre-clinical stage of drug discovery.

Regarding the possible mechanism of action of $14 \mathrm{e}$, the ability of furoxan derivatives to act as NO-donor compounds have been reported in the literature [64]. For compound $\mathbf{1 4 e}$, we have characterized its ability to release NO through in vitro assays [17]. During Leishmania infection, phagolysosomal amastigotes can decrease NO production in infected macrophages by inhibition of iNOS $[65,66]$. In fact, it was demonstrated that iNOS knockout mice are highly susceptible to Leishmania infection [67]. Since NO levels are reduced in infected macrophages, compounds with NO donor ability could help to eliminate intracellular amastigote forms of the parasite [17]. Herein, we showed that compound $14 \mathrm{e}$ is able to induce the NO production in macrophages. If the NO production was dependent of the compound itself, other derivatives (14a-14d) previously described as each having the potential to generate NO [17] should have demonstrated the same NO release as $14 \mathrm{e}$, but that was not observed. In addition, 14e might inhibit Leishmania cysteine protease, an important enzyme that allow the parasite to evade the host immune response [28], since the $N$-acyl hydrazone moiety presented in this molecule has been shown to inhibit this enzyme $[15,16]$. Thus, in order to optimize this interesting antileishmanial compound, we are currently evaluating if $14 \mathrm{e}$ is able to inhibit cysteine protease as well as working to determine through proteomic approaches their other molecular targets.

\section{Conclusion}

At this stage, our data point to the notion that compound 14e might be considered a promising drug for the treatment of visceral leishmaniasis, since this compound reduced the parasite burden in liver and spleen of $L$. infantum-infected golden hamsters. In addition, this compound did not cause toxicological effects at the effective dose $(3.0 \mathrm{mg} /$ $\mathrm{Kg}$ /day).

\section{Acknowledgments}

The authors thank the São Paulo Research Foundation (FAPESP) for financial support (grant \#2013/08248-1 and \#2017/03552-5), Coordenação de Aperfeiçoamento de Pessoal de Nível Superior (CAPES), Conselho Nacional de Desenvolvimento Científico e Tecnológico (CNPq - grant: 140452/2013-3) and PADC. LA, TGP and JNV were supported by CAPES and FAPESP fellowships. We are also grateful to Paul Michels for the critical reading of this article and Isabel Martinez for technical support.

\section{References}

[1] WHO, Control of Leishmaniasis, Expert Commitee, World Health Organization, 2010, pp. 1-186.

[2] B.S. McGwire, A.R. Satoskar, Leishmaniasis: clinical syndromes and treatment., QJM Mon, J. Assoc. Phys. 107 (2014) 7-14, http://dx.doi.org/10.1093/qjmed/ het116.

[3] M.P. Barrett, S.L. Croft, Management of trypanosomiasis and leishmaniasis, $\mathrm{Br}$. Med. Bull. 104 (2012) 175-196, http://dx.doi.org/10.1093/bmb/lds031.

[4] J. Alvar, S. Croft, P. Olliaro, Chemotherapy in the treatment and control of leishmaniasis, Adv. Parasitol. 61 (2006) 223-274, http://dx.doi.org/10.1016/S0065308X(05)61006-8.

[5] P. Kaye, P. Scott, Leishmaniasis: complexity at the host-pathogen interface, Nat. Rev. Microbiol. 9 (2011) 604-615, http://dx.doi.org/10.1038/nrmicro2608.

[6] S.L. Croft, P. Olliaro, Leishmaniasis chemotherapy-challenges and opportunities, Clin. Microbiol. Infect. 17 (2011) 1478-1483, http://dx.doi.org/10.1111/j.1469 0691.2011.03630.x.

[7] M. Den Boer, D. Argaw, J. Jannin, J. Alvar, Leishmaniasis impact and treatment access, Clin. Microbiol. Infect. 17 (2011) 1471-1477, http://dx.doi.org/10.1111/j. 1469-0691.2011.03635.x.

[8] S. Sundar, Drug resistance in indian visceral leishmaniasis, Trop. Med. Int. Heal. 6 (2001) 849-854, http://dx.doi.org/10.1046/j.1365-3156.2001.00778.x.

[9] N. Singh, M. Kumar, R.K. Singh, Leishmaniasis: current status of available drugs and new potential drug targets, Asian Pac. J. Trop. Med. 5 (2012) 485-497, http://dx. doi.org/10.1016/S1995-7645(12)60084-4.

[10] T.G. Passalacqua, L.A. Dutra, L. de Almeida, A.M.A. Velásquez, F.A.E. Torres, P.R. Yamasaki, M.B. dos Santos, L.O. Regasini, P.A.M. Michels, V.D.S. Bolzani, M.A.S. Graminha, Synthesis and evaluation of novel prenylated chalcone derivatives as anti-leishmanial and anti-trypanosomal compounds, Bioorg. Med. Chem. Lett. 25 (2015) 3342-3345, http://dx.doi.org/10.1016/j.bmcl.2015.05.072.

[11] V. dos Santos, K. Leite, M. da Costa Siqueira, L. Regasini, I. Martinez, C. Nogueira, M. Galuppo, B. Stolf, A. Pereira, R. Cicarelli, M. Furlan, M. Graminha, Antiprotozoal activity of quinonemethide triterpenes from Maytenus ilicifolia (Celastraceae), Molecules 18 (2013) 1053-1062, http://dx.doi.org/10.3390/molecules18011053.

[12] V.A. Santos, L.O. Regasini, C.R. Nogueira, G.D. Passerini, I. Martinez, V.S. Bolzani, M.A.S. Graminha, R.M.B. Cicarelli, M. Furlan, Antiprotozoal sesquiterpene pyridine alkaloids from Maytenus ilicifolia, J. Nat. Prod. 75 (2012) 991-995, http://dx.doi. org $/ 10.1021 / \mathrm{np} 300077 \mathrm{r}$.

[13] H. Cerecetto, W. Porcal, Pharmacological properties of furoxans and benzofuroxans: recent developments, Mini Rev. Med. Chem. 5 (2005) 57-71.

[14] D. Castro, L. Bioani, D. Benitez, P. Hérnandez, A. Merlino, C. Gil, D. Castro, L. Boiani, D. Benitez, P. Herna, H. Cerecetto, W. Porcal, C. Olea-azar, M. Gonza, Anti-trypanosomatid benzofuroxans and deoxygenated analogues: synthesis using 
polymer-supported triphenylphosphine, biological evaluation and mechanism of action studies, Eur. J. Med. Chem. 44 (2009) 5055-5065, http://dx.doi.org/10. 1016/j.ejmech.2009.09.009.

[15] L. Boiani, G. Aguirre, M. González, H. Cerecetto, A. Chidichimo, J.J. Cazzulo, M. Bertinaria, S. Guglielmo, Furoxan-, alkylnitrate-derivatives and related compounds as anti-trypanosomatid agents: mechanism of action studies, Bioorg. Med. Chem. 16 (2008) 7900-7907, http://dx.doi.org/10.1016/j.bmc.2008.07.077.

[16] P. Hernández, R. Rojas, R.H. Gilman, M. Sauvain, L.M. Lima, E.J. Barreiro, M. González, H. Cerecetto, Hybrid furoxanyl N-acylhydrazone derivatives as hits for the development of neglected diseases drug candidates, Eur. J. Med. Chem. 59 (2013) 64-74, http://dx.doi.org/10.1016/j.ejmech.2012.10.047.

[17] L.A. Dutra, L. de Almeida, T.G. Passalacqua, J.S. Reis, F.A.E. Torres, I. Martinez, R.G. Peccinini, C.M. Chin, K. Chegaev, S. Guglielmo, R. Fruttero, M.A.S. Graminha, J.L. dos Santos, Leishmanicidal activities of novel synthetic furoxan and benzofuroxan derivatives, Antimicrob. Agents Chemother. 58 (2014) 4837-4847, http:// dx.doi.org/10.1128/AAC.00052-14.

[18] H. Moshage, B. Kok, J.R. Huizenga, P.L.M. Jansen, Nitrite and nitrate determinations in plasma: a critical evaluation, Clin. Chem. 41 (1995) 892-896.

[19] L.C. Green, D.A. Wagner, J. Glogowski, P.L. Skipper, J.S. Wishnok, S.R. Tannenbaum, Analysis of nitrate nitrite, and [15N] nitrate in biological fluids, Anal. Biochem. 126 (1982) 131-138.

[20] L.C. Afonso, P. Scott, Immune responses associated with susceptibility of C57BL/10 mice to Leishmania amazonensis, Infect. Immun. 61 (1993) 2952-2959.

[21] H.C. Lima, J.A. Bleyenberg, R.G. Titus, A simple method for quantifying Leishmania in tissues of infected animals, Parasitol. Today 13 (1997) 80-82, http://dx.doi.org/ 10.1016/S0169-4758(96)40010-2.

[22] I.M. Washington, G. Van Hoosier, The Laboratory Rabbit, Guinea Pig, Hamster, and Other Rodents, Elsevier, 2012, http://dx.doi.org/10.1016/B978-0-12-380920-9. 00003-1.

[23] T.T.H. Pham, P.M. Loiseau, G. Barratt, Strategies for the design of orally bioavailable antileishmanial treatments, Int. J. Pharm. (2013) 1-14, http://dx.doi.org/10. 1016/j.ijpharm.2013.07.035.

[24] M.C. Chung, E.I. Ferreira, J.L. Santos, J. Giarolla, D.G. Rando, A.E. Almeida, P.L. Bosquesi, R.F. Menegon, L. Blau, Prodrugs for the treatment of neglected diseases, Molecules 13 (2007) 616-677, http://dx.doi.org/10.3390/ molecules13030616.

[25] U. Galli, L. Lazzarato, M. Bertinaria, G. Sorba, A. Gasco, S. Parapini, D. Taramelli, Synthesis and antimalarial activities of some furoxan sulfones and related furazans, Eur. J. Med. Chem. 40 (2005) 1335-1340, http://dx.doi.org/10.1016/j.ejmech. 2005.05.001.

[26] G. Rai, A.A. Sayed, W.A. Lea, H.F. Luecke, H. Chakrapani, S. Prast-nielsen, A. Jadhav, W. Leister, M. Shen, J. Inglese, C.P. Austin, L. Keefer, E.S.J. Arn, A. Simeonov, D.J. Maloney, D.L. Williams, C.J. Thomas, Structure mechanism insights and the role of nitric oxide donation guide the development of oxadiazole-2oxides as therapeutic agents against schistosomiasis, J. Med. Chem. 52 (2009) 6474-6483, http://dx.doi.org/10.1021/jm901021k.

[27] M. Bertinaria, S. Guglielmo, B. Rolando, M. Giorgis, C. Aragno, R. Fruttero, A. Gasco, S. Parapini, D. Taramelli, Y.C. Martins, L.J.M. Carvalho, Amodiaquine analogues containing NO-donor substructures: synthesis and their preliminary evaluation as potential tools in the treatment of cerebral malaria, Eur. J. Med. Chem. 46 (2011) 1757-1767, http://dx.doi.org/10.1016/j.ejmech.2011.02.029.

[28] M. Sajid, J.H. McKerrow, Cysteine proteases of parasitic organisms, Mol. Biochem. Parasitol. 120 (2002) 1-21, http://dx.doi.org/10.1016/S0166-6851(02)00043-9.

[33] E.E. Zijlstra, PKDL and other dermal lesions in HIV co-infected patients with leishmaniasis: review of clinical presentation in relation to immune responses, PLoS Negl. Trop. Dis. 8 (2014) 1-12, http://dx.doi.org/10.1371/journal.pntd.0003258.

[34] C.M. Espitia, O.A. Saldarriaga, B.L. Travi, E. Osorio, A. Hernandez, M. Band, M.J. Patel, A.A. Medina, M. Cappello, A. Pekosz, P.C. Melby, Transcriptional profiling of the spleen in progressive visceral leishmaniasis reveals mixed expression of type 1 and type 2 cytokine-responsive genes, BMC Imunol. 15 (2014), http://dx.doi. org/10.1186/s12865-014-0038-Z.

[35] M.Z. Wang, X. Zhu, A. Srivastava, Q. Liu, J.M. Sweat, T. Pandharkar, C.E. Stephens, E. Riccio, T. Parman, M. Munde, S. Mandal, R. Madhubala, R.R. Tidwell, W.D. Wilson, D.W. Boykin, J.E. Hall, D.E. Kyle, K.A. Werbovetz, Novel arylimidamides for treatment of visceral leishmaniasis, Antimicrob. Agents Chemother. 54 (2010) 2507-2516, http://dx.doi.org/10.1128/AAC.00250-10.

[36] C.M. Espitia, W. Zhao, O. Saldarriaga, Y. Osorio, L.M. Harrison, M. Cappello, B.L. Travi, P.C. Melby, Duplex real-time reverse transcriptase PCR to determine cytokine mRNA expression in a hamster model of New World cutaneous leishmaniasis, BMC. Immunol. 11 (2010), http://dx doi.org/10.1186/1471-2172-11-31.

[37] M.A. Dea-Ayuela, S. Rama-Íñiguez, J.M. Alunda, F. Bolás-Fernandez, Setting new immunobiological parameters in the hamster model of visceral leishmaniasis for in vivo testing of antileishmanial compounds, Vet. Res. Commun. 31 (2007) 703-717, http://dx.doi.org/10.1007/s11259-007-0040-5.

[38] M. Sharma, K. Chauhan, R. Shivahare, P. Vishwakarma, M.K. Suthar, A. Sharma, S. Gupta, J.K. Saxena, J. Lal, P. Chandra, B. Kumar, P.M.S. Chauhan, Discovery of a new class of natural product-inspired quinazolinone hybrid as potent antileishmanial agents, J. Med. Chem. 56 (2013) 4374-4392, http://dx.doi.org/10.1021/ jm400053v.

[39] J.A. Jesus, T.N. Fragoso, E.S. Yamamoto, M.D. Laurenti, M.S. Silva, A.F. Ferreira, J.H.G. Lago, G.S. Gomes, L.F.D. Passero, Therapeutic effect of ursolic acid in experimental visceral leishmaniasis, Int. J. Parasitol. Drugs Drug Resist. 7 (2017) 1-11, http://dx.doi.org/10.1016/j.ijpddr.2016.12.002.
[40] P.C. Melby, V.V. Tryon, B. Chandrasekar, G.L. Freeman, Cloning of syrian hamster (Mesocricetus auratus) cytokine cDNAs and analysis of cytokine mRNA expression in experimental visceral leishmaniasis, Infect. Immun. 66 (1998) 2135-2142.

[41] V. Rodrigues Jr., J. Santana da Silva, A. Campos-Neto, Transforming growth factor beta and immunosuppression in experimental visceral leishmaniasis, Infect. Immun. 66 (1998) 1233-1236.

[42] M.A. Miller, Reversible hepatoxicity related to amphotericin B, Can. Med. Assoc. J. 131 (1984) 1245-1247.

[43] I.A. El Hag, F.A. Hashim, I.A. El Toum, M. Homeida, Liver morphology and function in visceral leishmaniasis (Kala-azar), J. Clin. Phatology. 47 (1994) 547-551.

[44] M.E. Wilson, D.J. Innes, A.D. Sousa, R.D. Pearson, Early histopathology of experimental infection with Leishmania donovani in hamsters, J. Parasitol. 73 (1987) 55-63.

[45] V.L.P. Vianna, C. Takiya, L. de Brito-Gitirana, Histopathologic analysis of hamster hepatocytes submitted to experimental infection with Leishmania donovani, Parasitol. Res. 88 (2002) 829-836, http://dx.doi.org/10.1007/s00436-001-0577-0.

[46] M.D. Laurenti, M.N. Sotto, C.E. Corbett, V.L. da Matta, M.I. Duarte, Experimental visceral leishmaniasis: sequential events of granuloma formation at subcutaneous inoculation site, Int. J. Exp. Pathol. 71 (1990) 791-797.

[47] A. Gomes-Silva, J.G. Valverde, R.P. Ribeiro-Romão, R.M. Plácido-Pereira, A.M. DaCruz, Golden hamster (Mesocricetus auratus) as an experimental model for Leishmania (Viannia) braziliensis infection, Parasitology 140 (2013) 771-779, http://dx.doi.org/10.1017/S0031182012002156.

[48] W.J. Willians, D. Willians, The properties and development of conchoidal bodies in sarcoid and sarcoid-like granulomas, J. Pathol. Bacteriol. 96 (1968) 491-496.

[49] M. Cesta, Normal structure, function, and histology of the spleen, Toxicol. Pathol. 34 (2006) 455-465, http://dx.doi.org/10.1080/01926230600867743.

[50] A.C. Stanley, C.R. Engwerda, Balancing immunity and pathology in visceral leishmaniasis, Immunol. Cell Biol. 85 (2007) 138-147, http://dx.doi.org/10.1038/sj. icb7100011.

[51] B. Veress, A. Omer, A.A. Satir, A.M. El Hassan, Morphology of the spleen and lymph nodes in fatal visceral leishmaniasis, Immunology 33 (1977) 605-610.

[52] C.R. Engwerda, M. Ato, P.M. Kaye, Macrophages, pathology and parasite persistence in experimental visceral leishmaniasis, Trends Parasitol. 20 (2004) 524-530, http://dx.doi.org/10.1016/j.pt.2004.08.009.

[53] V. Rodrigues, A. Cordeiro-da-Silva, M. Laforge, R. Silvestre, J. Estaquier, Regulation of immunity during visceral Leishmania infection, Parasit. Vectors 9 (2016), http:// dx.doi.org/10.1186/s13071-016-1412-x.

[54] J.P. Botero Aguirre, A.M. Restrepo Hamid, Amphotericin B deoxycholate versus liposomal amphotericin B: effects on kidney function, Cochrane Database Syst. Rev. (2015) 1-60, http://dx.doi.org/10.1002/14651858.CD010481.pub2.

[55] R.J. Hamill, Amphotericin B formulations: a comparative review of efficacy and toxicity, Drugs 73 (2013) 919-934, http://dx.doi.org/10.1007/s40265-0130069-4.

[56] H. Khalili, S. Bairami, M. Kargar, Antibiotics induced acute kidney injury: incidence, risk factors, onset time and outcome, Acta Med. Iran 51 (2013) 871-878.

[57] R. Laniado-Laborín, M.N. Cabrales-Vargas, Amphotericin B: side effects and toxicity, Rev. Iberoam. Micol. 26 (2009) 223-227, http://dx.doi.org/10.1016/j.riam. 2009.06.003.

[58] F.F. Tuon, F. Koenig, D. Jacometto, J.L. Rocha, Are there risk factors for acute renal failure in adult patients using deoxycholate amphotericin B? Rev. Iberoam. Micol. 30 (2013) 21-24, http://dx.doi.org/10.1016/j.riam.2012.09.003.

[59] R.L. Wade, P. Chaudhari, J.L. Natoli, R.J. Taylor, B.H. Nathanson, D.L. Horn, Nephrotoxicity and other adverse events among inpatients receiving liposomal amphotericin B or amphotericin B lipid complex, Diagn. Microbiol. Infect, Dis . 76 (2013) 361-367, http://dx.doi.org/10.1016/j.diagmicrobio.2013.04.001.

[60] D.F. Bes, M.T. Rosanova, N. Sberna, E. Arrizurieta, Deoxycholate amphotericin B and nephrotoxicity in the pediatric setting, Pediatr. Infect. Dis. J. 33 (2014) 198-206, http://dx.doi.org/10.1097/INF.0000000000000299.

[61] S.S. Waikar, J.V. Bonventre, Creatinine kinetics and the definition of acute kidney injury, J. Am. Soc. Nephrol. 20 (2009) 672-679, http://dx.doi.org/10.1681/ASN. 2008070669.

[62] S.S. Waikar, R.A. Betensky, J.V. Bonventre, Creatinine as the gold standard for kidney injury biomarker studies? Nephrol. Dial. Transplant. 24 (2009) 3263-3265, http://dx.doi.org/10.1093/ndt/gfp428.

[63] S.S. Waikar, R.A. Betensky, S.C. Emerson, J.V. Bonventre, Imperfect gold standards for kidney injury biomarker evaluation, J. Am. Soc. Nephrol. 23 (2012) 13-21, http://dx.doi.org/10.1681/ASN.2010111124.

[64] P. Ascenzi, A. Bocedi, M. Gentile, P. Visca, L. Gradoni, Inactivation of parasite cysteine proteinases by the NO-donor 4-(phenylsulfonyl)-3-((2-(dimethylamino) ethyl)thio)-furoxan oxalate, Biochim. Biophys. Acta 2004 (1703) 69-77, http://dx. doi.org/10.1016/j.bbapap.2004.09.027

[65] F.M. Perrella Balestieri, A.R. Pires Queiroz, C. Scavone, V.M. Assis Costa, M. BarralNetto, I. de A. Abrahamsohn, Leishmania (L.) amazonensis-induced inhibition of nitric oxide synthesis in host macrophages, Microbes Infect. 4 (2002) 23-29, http:// dx.doi.org/10.1016/S1286-4579(01)01505-2.

[66] C. Bogdan, M. Röllinghoff, How do protozoan parasites survive inside macrophages? Parasitol. Today 15 (1999) 22-28, http://dx.doi.org/10.1016/S01694758(98)01362-3.

[67] X.Q. Wei, I.G. Charles, A. Smith, J. Ure, G.J. Feng, F.P. Huang, D. Xu, W. Muller, S. Moncada, F.Y. Liew, Altered immune responses in mice lacking inducible nitric oxide synthase, Nature 375 (1995) 408-411, http://dx.doi.org/10.1038/375408a0. 